Article

\title{
TBAB-Catalyzed 1,6-Conjugate Sulfonylation of para- Quinone Methides: A Highly Efficient Approach to Unsymmetrical gem-Diarylmethyl Sulfones in Water ${ }^{\dagger}$
}

\author{
Zhang-Qin Liu *(D), Peng-Sheng You, Liang-Dong Zhang, Da-Qing Liu, Sheng-Shu Liu and \\ Xiao-Yu Guan * \\ Key Laboratory of Applied Chemistry of Chongqing Municipality, College of Chemistry and Chemical \\ Engineering, Southwest University, Chongqing 400715, China \\ * Correspondence: liuzq999@swu.edu.cn (Z.-Q.L.); guanxiaoyu@swu.edu.cn (X.-Y.G.); \\ Tel.: +86-023-68252360 (Z.-Q.L. \& X.-Y.G.) \\ + Dedicated to Professor Jean-Marie Basset on the occasion of his 77th birthday.
}

Academic Editor: Georg Manolikakes

Received: 29 December 2019; Accepted: 24 January 2020; Published: 26 January 2020

\begin{abstract}
A highly efficient sulfonylation of para-quinone methides with sulfonyl hydrazines in water has been developed on the basis of the mode involving a tetrabutyl ammonium bromide (TBAB)-promoted sulfa-1,6-conjugated addition pathway. This reaction provides a green and sustainable method to synthesize various unsymmetrical diarylmethyl sulfones, showing good functional group tolerance, scalability, and regioselectivity. Further transformation of the resulting diarylmethyl sulfones provides an efficient route to some functionalized molecules.
\end{abstract}

Keywords: sulfa-1,6-conjugated addition; unsymmetrical diarylmethyl sulfones; green and sustainable chemistry; synthesis in water

\section{Introduction}

As a class of sulfur-containing compounds, sulfones are widely used in organic synthesis, pharmaceuticals, agrochemicals, and materials science [1-7]. Among the sulfone family, diarylmethyl sulfones hold an essential position in biologically important compounds that show various biological activities, such as potassium channel inhibitory activity [8], as well as antidepressant [9] and anticancer properties (Figure 1) [10]. Additionally, diarylmethyl sulfones can be applied as useful intermediates for synthetic applications owing to the versatile reactivities of the sulfonyl group activated carbanions [1,11-14].
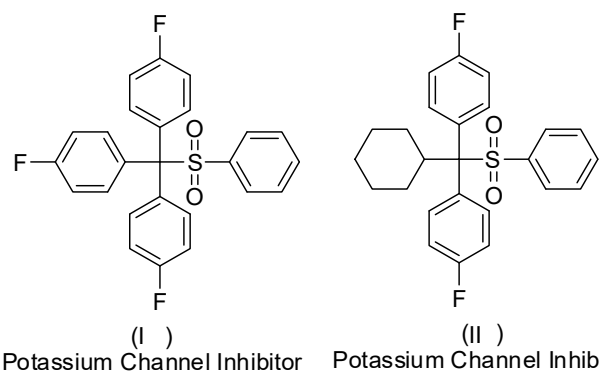

(II)

Potassium Channel Inhibitor

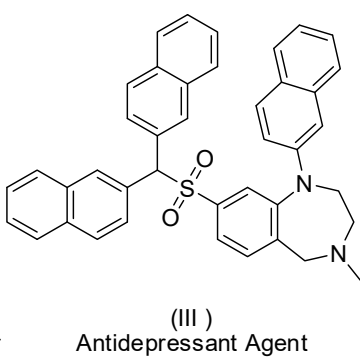

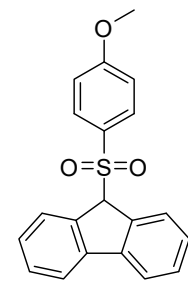

(IV)

Anticancer Agent

Figure 1. Representative biologically active diarylmethyl sulfone derivatives.

Although a number of methodologies have been developed for the synthesis of diarylmethyl sulfones, most of the reported methods suffer from the harsh reaction conditions, multi-step procedures, 
and requirements of expensive metal catalysts or potentially toxic organic solvents [15-18], which make them unsustainable and environmentally unfavorable.

Concerns about the environmental issues caused by the influence of human society have currently become prevalent and ubiquitous. Engagement on green and sustainable methods of chemical synthesis has emerged as a pioneering realm [19-23] garnering of enormous attention of chemists and biologists. Among them, chemical reactions "in water" [24] offered a novel way to green and sustainable synthesis. Water, compared to the traditional organic solvents, possesses distinctive properties such as safety, innocuousness, high heat capacity, extensive hydrogen bonding, and redox stability [24-26]. Consequently, water has been gradually accepted [27-33] as a desirable reaction medium since the first water-promoted Diels-Alder reaction reported by Breslow [34] in 1980.

The C-S bond formation reaction is still an intriguing field attributed to the versatile block-building usages $[35,36]$ and the bioactive agents $[37,38]$ of sulfur-containing compounds. Thus, substantial endeavors have been focused on the achievement of the efficient, expedient, and low-cost approach. With our keen interest in the sulfa-1,6-conjugated addition reaction $[15,39,40]$, we try to combine the green and sustainable chemical synthetic concept and the C-S bonding formation reactions. Herein, we disclose a highly efficient tetrabutyl ammonium bromide (TBAB)-promoted sulfonylation of para-quinone methide $(p-\mathrm{QM})$ with sulfonyl hydrazines to afford unsymmetrical gem-diarylmethyl sulfones via a sulfa-1,6-conjugated addition pathway. All these reactions were performed smoothly in water under mild conditions (Scheme 1).

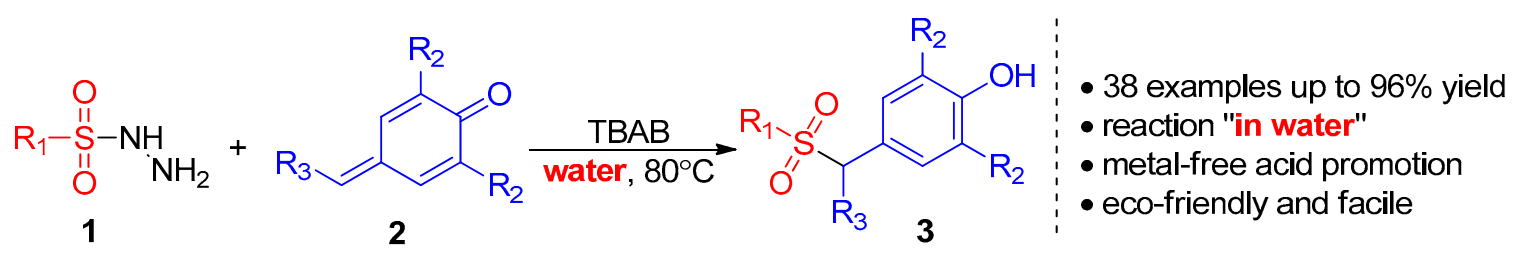

Scheme 1. Synthesis of unsymmetrical gem-diarylmethyl sulfones via sulfa-1,6-conjugated addition in water.

\section{Results and Discussion}

\subsection{Optimization of Reaction Conditions}

In our preliminary study, the investigation of the reaction conditions was carried out with 4-methylbenzenesulfonohydrazide 1a and para-quinone methides ( $p-Q M)$ 2a as model substrates (Table 1). Initially, the reaction was explored with different solvents in absence of a catalyst. When the reaction was carried out at $80^{\circ} \mathrm{C}$ in tetrahydrofuran (THF), the desired 1,6-sulfa-conjugated adduct was obtained in mediate yield (50\%). Other organic solvents including $\mathrm{Et}_{2} \mathrm{O}$, toluene, dichloroethane (DCE), dioxane, and dimethylsulfoxide (DMSO) were proved to be unsuitable for the reaction (Table 1, entry 1-6). It was found that protic polar solvent had an obvious promotion effect on the reaction. When $\mathrm{H}_{2} \mathrm{O}$ and $\mathrm{EtOH}$ were employed, the yield was increased slightly to $61 \%$ and $73 \%$, respectively (Table 1, entry 7-8). Although EtOH had a better effect than water, taking into consideration the "green and sustainable chemistry" perspective, we chose $\mathrm{H}_{2} \mathrm{O}$ as a solvent. Subsequently, we focused on catalyst screening to improve the yield of the water mediated C-S bond formation reaction. Unfortunately, heterogeneous transition metal nano-catalysts such as $\mathrm{Pd} / \mathrm{C}, \mathrm{Pd} / \mathrm{TiO}{ }_{2}, \mathrm{Au} / \mathrm{TiO}{ }_{2}$, and $\mathrm{Pt} / \mathrm{C}$ had little promotion effect on the reaction (Table 1, entry 9-12). Inspired by the better result of $\mathrm{EtOH}$, phase transfer catalysts were selected to improve the solubility of organic substrate 1a and $2 \mathrm{a}$ in water, which may have a positive influence on the reaction (Table 1, entry 13-17). To our delight, in presence of $10 \%$ mol tetrabutylammonium bromide (TBAB), the yield was improved sharply to $82 \%$ (Table 1, entry 16). Based on the result, we continuously investigated the effect of other reaction parameters including temperature, reaction time, and reactant ratio. We were pleased to find that the yield was markedly increased, as the reactant ratio of 2a:1a was increased from 1 to 1.5. Thus, the 
desired gem-diarylmethyl sulfone 3aa was obtained in 96\% yield by performing the TBAB-catalyzed sulfonylation of 4-methylbenzenesulfonohydrazide 1a to $p$-QM 2a at $80{ }^{\circ} \mathrm{C}$ in $12 \mathrm{~h}$ (Table 1, entry 22). Given either a lower or higher temperature, the yields decreased (Table 1, entry 18-20).

Table 1. Optimization of the reaction conditions for the 1,6-conjugate sulfonylation of 4-methylbenzenesulfonohydrazide $1 \mathbf{a}$ to $p-Q M 2 \mathbf{a}^{\mathrm{a}}$.

\begin{tabular}{cccccc} 
& & & & \\
\hline 1a & & & & & \\
\hline
\end{tabular}

a The reaction conditions: $\mathbf{1 a}(0.20 \mathrm{mmol}), \mathbf{2 a}(0.20 \mathrm{mmol})$, catalyst $(0.02 \mathrm{mmol})$, solvent $(1 \mathrm{~mL}),{ }^{\mathrm{b}}$ isolated yield based on 1a, ${ }^{\mathrm{C}} \mathbf{2 a}(0.3 \mathrm{mmol})$.

\subsection{Reaction Scope}

Based on the optimized reaction conditions, the generality of this 1,6-conjugate sulfonylation reaction of sulfonyl hydrazines to para-quinone methides was investigated. Some of the results are summarized in Figures 2 and 3.

A number of para-quinone methide derivatives 2 bearing different substituents were explored with 4-methylbenzenesulfonohydrazide 1a under standard reaction condition (Figure 2). Various substituent groups, such as electron-donating groups (-Me, -OMe), electron-withdrawing groups $\left(-\mathrm{CF}_{3},-\mathrm{NO}_{2}\right)$, and halogen atoms $(\mathrm{F}, \mathrm{Cl}, \mathrm{Br})$, on the $p$-QM derivatives' aryl ring at the ortho (2ab-2ag), meta (2ah-2al), and para (2am-2as) positions were well tolerated by the reaction to provide the corresponding sulfonylation adducts in good to excelled yields. Furthermore, non-substituted phenyl substrate 2aa, disubstituted 2,4-dichlorophenyl substrate 2at, polycyclic aromatic substrate 2av, heteroaromatic substrates 2au, and aliphatic substrate 2ay led to the desired product in 63-95\% yields, implying the well-tolerated property of the substrates in this reaction. Moreover, $p$-QMs derived from 2,6-dimethylphenol 2aw and 2,6-diisopropylphenol 2ax were also compatible to afford the 
corresponding adducts, while the former was in lower yield (3aw, 63\%) probably due to its small steric hindrance around the phenolic hydroxyl group.

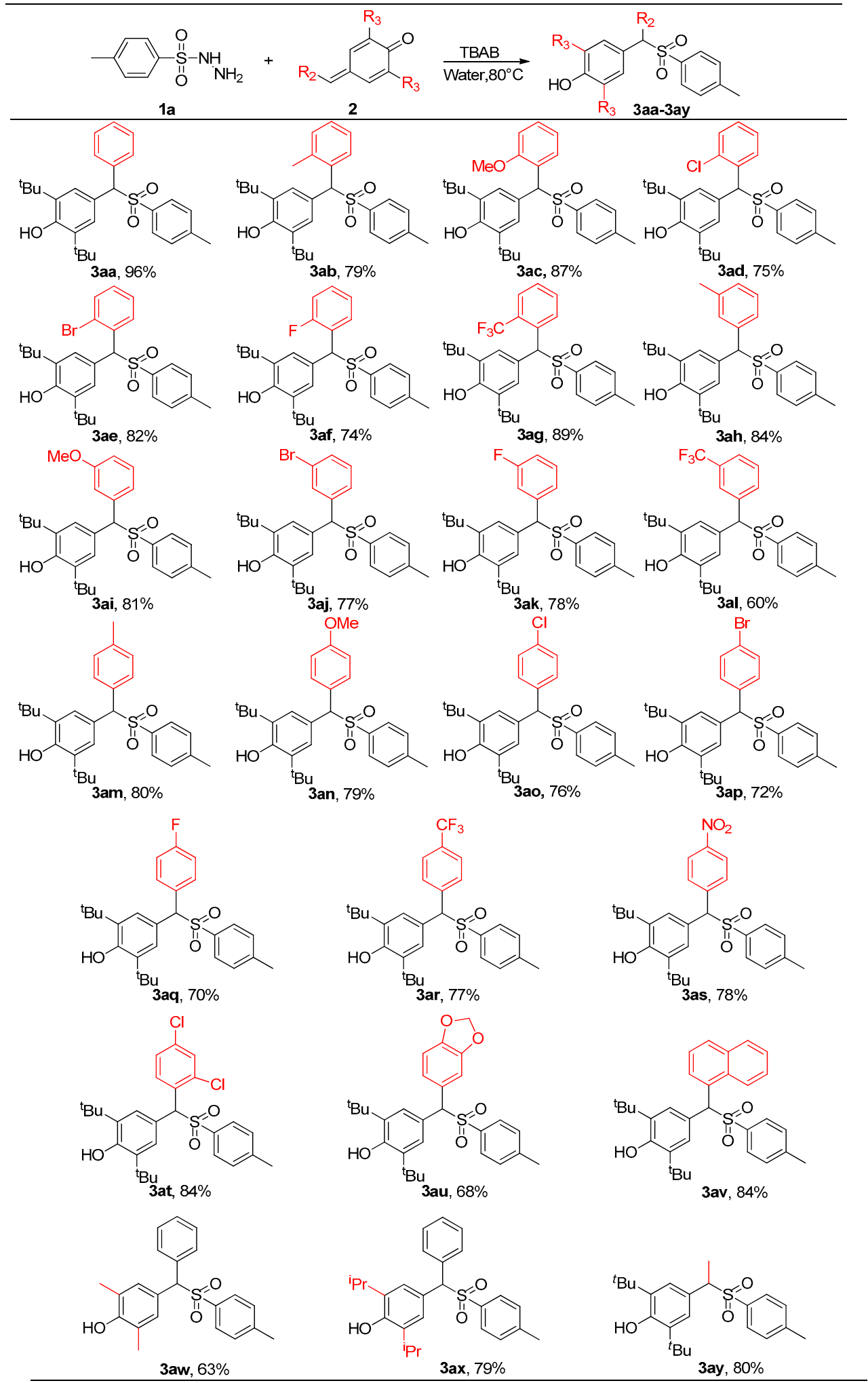

Figure 2. Tetrabutylammonium bromide (TBAB)-catalyzed 1,6-conjugate sulfonylation for synthesis of gem-diarylmethyl sulfones 3aa-3ay. ${ }^{a}$ The reaction conditions: sulfonyl hydrazides $1 \mathbf{1 a}(0.20 \mathrm{mmol})$, p-QM $2(0.30 \mathrm{mmol})$, TBAB $(0.02 \mathrm{mmol})$ in $1 \mathrm{~mL}$ of $\mathrm{H}_{2} \mathrm{O}, 80^{\circ} \mathrm{C}, 12 \mathrm{~h},{ }^{\mathrm{b}}$ Isolated yield base on $1 \mathrm{a}$. 


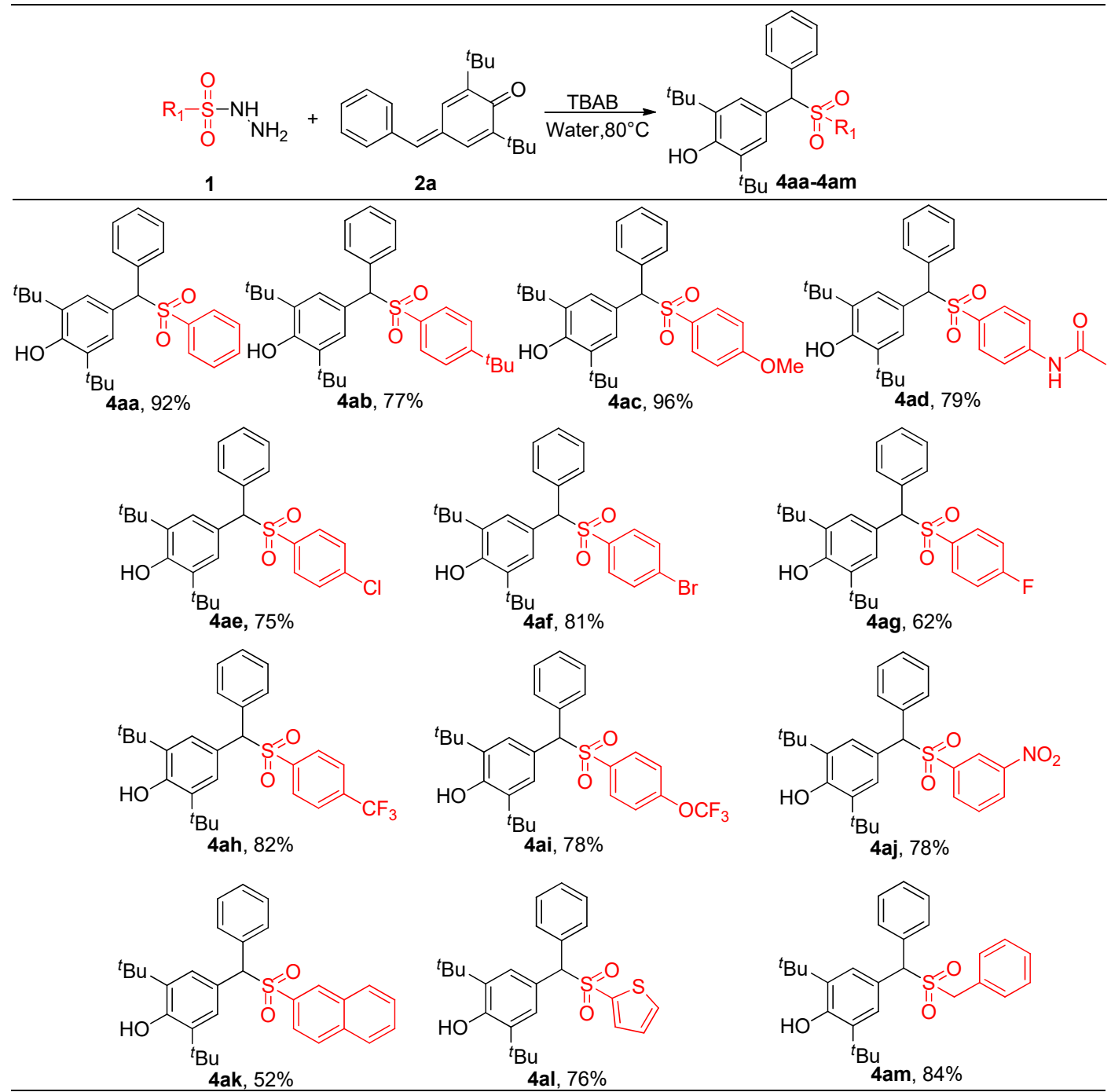

Figure 3. TВAB catalyzed 1,6-conjugate sulfonylation for synthesis of gem-diarylmethyl sulfones 4aa-4am. ${ }^{a}$ The reaction conditions: sulfonyl hydrazides $\mathbf{1}(0.20 \mathrm{mmol})$, para-quinone methide $(p-\mathrm{QM})$ 2a $(0.30 \mathrm{mmol}), \operatorname{TBAB}(0.02 \mathrm{mmol})$ in $1 \mathrm{~mL}$ of $\mathrm{H}_{2} \mathrm{O}, 80^{\circ} \mathrm{C}, 12 \mathrm{~h}, \mathrm{~b}$ isolated yield base on 1.

Consequentially, we investigated the substitute effects of sulfonyl hydrazines by using 4-benzylidene-2,6-di-tert-butylcyclohexa-2,5-dienone $\mathbf{2 a}$ as model substrate under optimized reaction condition (Figure 3). In general, a wide range of sulfonyl hydrazines 1 bearing meta and para substituent group including electron-donating groups (-Me, - $\left.\mathrm{OMe},-\mathrm{NHAc},-\mathrm{OCF}_{3}\right)$, electron-withdrawing groups $\left(-\mathrm{CF}_{3},-\mathrm{NO}_{2}\right)$, and halogen atom $(\mathrm{F}, \mathrm{Cl}, \mathrm{Br})$ reacted smoothly with $p$-QM $2 \mathrm{a}$, affording the adducts in $62-96 \%$ yields. In addition, phenyl 4aa, $p$-tert-butyl phenyl 4ab, 2-naphthyl 4ak, thienyl $4 a$ and aliphatic benzyl substituted sulfonyl hydrazines were also tolerated by the reaction to produce the final products in $52-92 \%$ yields.

\subsection{Proposed Mechanism}

The chemical structure of diarylmethyl sulfones $\mathbf{3}$ and $\mathbf{4}$ were characterized by nuclear magnetic resonance spectroscopies (Supplementary Materials). Because there is another possible sulfinic ester adduct, the two potential products cannot be distinguished by NMR. To further identify the structure of the products, 4af was selected as a representative compound, and the sulfone structure was unequivocally confirmed by single crystal X-ray diffraction analysis, as shown in Figure 4 (CCDC No. 1531154). 

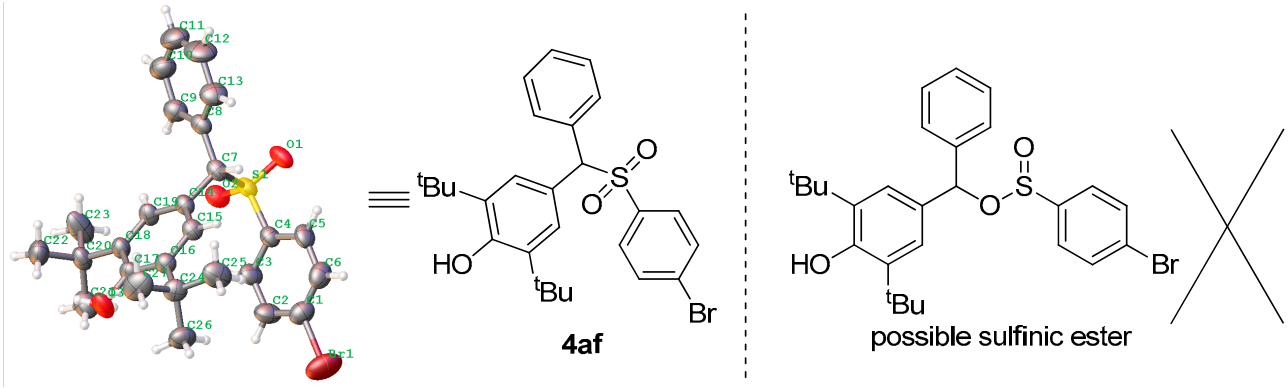

Figure 4. Crystal structure of diarylmethyl sulfone 4af and possible sulfinic ester.

On the basis of the above observations, we tentatively propose a plausible reaction mechanism (Scheme 2). First, the sulfonyl hydrazines 1 decomposed into sulfinyl anion 5 with the $\mathrm{N}_{2}$ released and hydronium generation under the heating condition. Intermediate 5 has a resonate equilibrium with sulfur-centered anion $\mathbf{6}$ in water. As a more reactive species, the sulfur-centered anion $\mathbf{6}$ attacks the electrophilic atom of the resonated structure of $p$-QM 2 with the help of tetrabutylammonium bromide (TBAB). Finally, driven by the aromatization force, the target product is obtained.

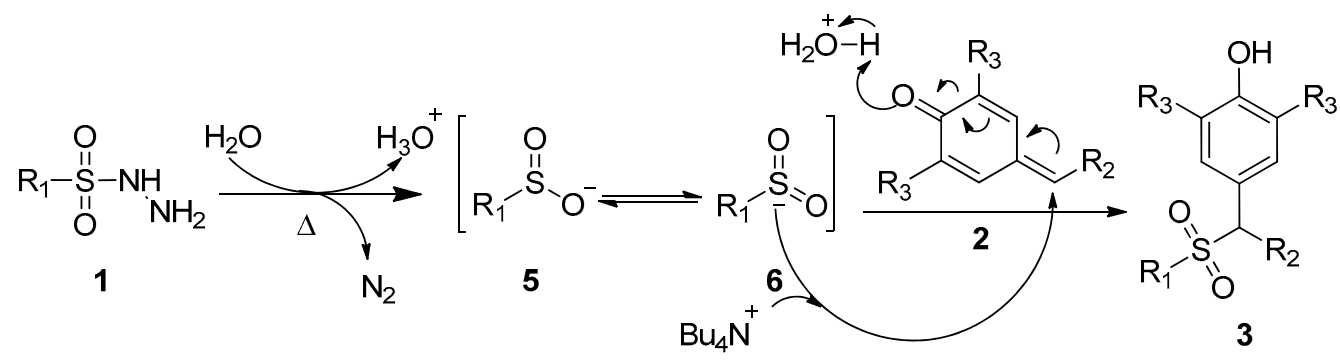

Scheme 2. Probable mechanism of the sulfa-1,6-conjugate reactions in water.

\subsection{Derivatives of Products}

To further demonstrate the synthetic utility of this protocol, the transformations of products were then explored (Scheme 3). A carbon-carbon bond formation between 3aa and indole led to the generation of unsymmetrical triarylmethane 7. Furthermore, base-promoted carbon-sulfur bond formation between 3aa and thiophenol proceeded to the facial delivery of unsymmetrical gem-diarylmethyl thioether 8. Moreover, the yield of products of both synthesis routines reached around $80 \%$, implying the potential and feasibility of further application of 3aa.

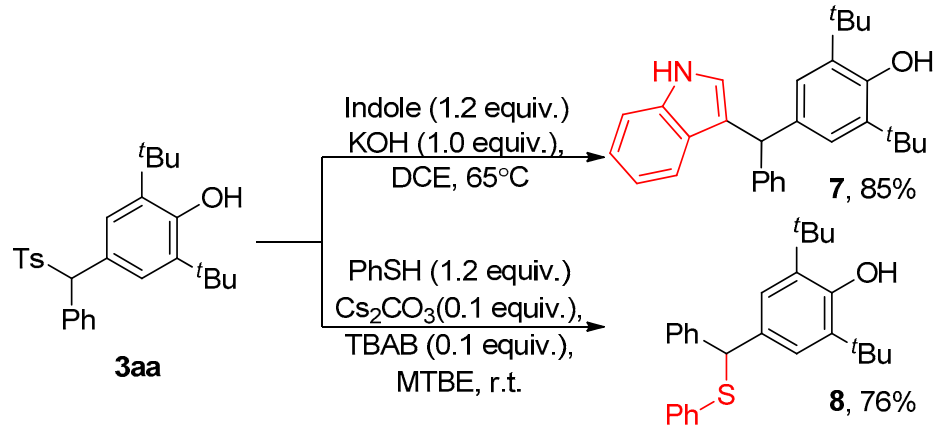

Scheme 3. Synthetic utility of diarylmethyl sulfone 3aa. 


\section{Materials and Methods}

\subsection{General Information}

${ }^{1} \mathrm{H}-\mathrm{NMR},{ }^{13} \mathrm{C}-\mathrm{NMR}$ spectra were obtained utilizing a Bruker 600 and $400 \mathrm{MHz}$ instrument and reported in $\mathrm{CDCl}_{3}$ or $\mathrm{DMSO}\left(\mathrm{d}^{6}\right)$. ${ }^{1} \mathrm{H}$ and ${ }^{13} \mathrm{CNMR}$ chemical shifts are reported in ppm relative to either TMS $\left({ }^{1} \mathrm{H}\right)(\delta=0 \mathrm{ppm})$ as an internal standard or the residual solvent peak as following: $\mathrm{CDCl}_{3}=7.26$ $\left({ }^{1} \mathrm{H}-\mathrm{NMR}\right),\left(\mathrm{CD}_{3}\right)_{2} \mathrm{SO}=2.50\left({ }^{1} \mathrm{H}-\mathrm{NMR}\right), \mathrm{CDCl}_{3}=77.16\left({ }^{13} \mathrm{C}-\mathrm{NMR}\right),\left(\mathrm{CD}_{3}\right)_{2} \mathrm{SO}=40.00\left({ }^{13} \mathrm{C}-\mathrm{NMR}\right)$. HRMS were performed on a Bruker Impact II 10200 instrument. Commercially available chemicals and solvents were purchased from Adamas-beta, Energy Chemical, Chongqing Chuandong Chemical, and Chengdu Kelong Chemical. The corresponding compounds were synthesized according to the methods reported in the literature. Analytical thin-layer chromatography (TLC) was performed on silicycle silica gel plates with F-254 indicator, and compounds were visualized by irradiation with UV light. Chromatography was carried out using silica gel 300-400 mesh.

\subsection{Experiment}

\subsubsection{Representative Procedure for Synthesis of Gem-Diarylmethyl Sulfones}

To a solution of corresponding 4-(arylmethylidene)-2,6-di-tert-butylcyclohexa-2,5-dienone $(0.3 \mathrm{mmol})$ in $1 \mathrm{~mL}$ water, the corresponding arylsulfonohydrazide $(0.2 \mathrm{mmol})$ and TBAB $(0.02 \mathrm{mmol})$ were added. The mixture was stirred at $80^{\circ} \mathrm{C}$. The reaction was monitored by TLC. After complete reaction, the mixture was extracted by ethyl acetate, dried over by anhydrous magnesium sulfate, and concentrated in vacuo. The crude product was then purified by flash column chromatography on silica gel (gradient eluent of PE/EA = 30:1-10:1) to gain the corresponding product.

2,6-Di-tert-butyl-4-(phenyl(tosyl)methyl)phenol (3aa): [15] Pale yellow solid; 96\% yield. ${ }^{1} \mathrm{H}-\mathrm{NMR}(600 \mathrm{MHz}$, $\left.\mathrm{CDCl}_{3}\right) \delta 7.61(\mathrm{~d}, J=7.1 \mathrm{~Hz}, 2 \mathrm{H}), 7.42(\mathrm{~d}, J=8.0 \mathrm{~Hz}, 2 \mathrm{H}), 7.32(\mathrm{dq}, J=14.2,6.9 \mathrm{~Hz}, 3 \mathrm{H}), 7.16(\mathrm{~s}, 2 \mathrm{H})$, $7.13(\mathrm{~d}, J=7.9 \mathrm{~Hz}, 2 \mathrm{H}), 5.20(\mathrm{~s}, 1 \mathrm{H}), 5.16(\mathrm{~s}, 1 \mathrm{H}), 2.36(\mathrm{~s}, 3 \mathrm{H}), 1.35(\mathrm{~s}, 18 \mathrm{H}) ;{ }^{13} \mathrm{C}-\mathrm{NMR}\left(151 \mathrm{MHz}, \mathrm{CDCl}_{3}\right)$ $\delta 154.08$ (s), 144.00 (s), 135.94 (s), 135.77 (s), 133.66 (s), 130.00 (s), 129.10 (s), 129.05 (s), 128.62 (s), 128.36 (s), 127.10 (s), 123.57 (s), 76.90 (s), 34.31 (s), 30.16 (s), 21.48 (s).

2,6-Di-tert-butyl-4-(o-tolyl(tosyl)methyl)phenol (3ab): [15] Pale yellow solid; 76\% yield. ${ }^{1} \mathrm{H}-\mathrm{NMR}(600 \mathrm{MHz}$, $\left.\mathrm{CDCl}_{3}\right) \delta 8.23(\mathrm{~d}, J=7.8 \mathrm{~Hz}, 1 \mathrm{H}), 7.44(\mathrm{~d}, J=8.0 \mathrm{~Hz}, 2 \mathrm{H}), 7.30(\mathrm{t}, J=7.5 \mathrm{~Hz}, 1 \mathrm{H}), 7.19(\mathrm{t}, J=7.4 \mathrm{~Hz}, 1 \mathrm{H})$, $7.14(\mathrm{~d}, J=8.0 \mathrm{~Hz}, 2 \mathrm{H}), 7.13(\mathrm{~s}, 2 \mathrm{H}), 7.08(\mathrm{~d}, J=7.5 \mathrm{~Hz}, 1 \mathrm{H}), 5.43(\mathrm{~s}, 1 \mathrm{H}), 5.21(\mathrm{~s}, 1 \mathrm{H}), 2.37(\mathrm{~s}, 3 \mathrm{H}), 2.17(\mathrm{~s}$, 3H), 1.34 (s, 18H). ${ }^{13} \mathrm{C}-\mathrm{NMR}\left(151 \mathrm{MHz}, \mathrm{CDCl}_{3}\right) \delta 153.99$ (s), 143.87 (s), 138.20 (s), 135.84 (s), 130.49 (s), 129.84 (s), 129.33 (s), 129.09 (s), 128.99 (s), 127.03 (s), 123.75 (s), 76.67 (s), 34.28 (s), 30.13 (s), 21.47 (s), 21.09 (s).

2,6-Di-tert-butyl-4-((2-methoxyphenyl)(tosyl)methyl)phenol (3ac): [15] Pale yellow solid; 87\% yield. ${ }^{1} \mathrm{H}-\mathrm{NMR}\left(600 \mathrm{MHz}, \mathrm{CDCl}_{3}\right) \delta 8.14(\mathrm{~d}, J=7.6 \mathrm{~Hz}, 1 \mathrm{H}), 7.44(\mathrm{~d}, J=7.9 \mathrm{~Hz}, 2 \mathrm{H}), 7.22-7.25(\mathrm{~m}$, $3 \mathrm{H}), 7.13(\mathrm{~d}, J=7.8 \mathrm{~Hz}, 2 \mathrm{H}), 7.04(\mathrm{t}, J=7.5 \mathrm{~Hz}, 1 \mathrm{H}), 6.73(\mathrm{~d}, J=8.2 \mathrm{~Hz}, 1 \mathrm{H}), 5.93(\mathrm{~s}, 1 \mathrm{H}), 5.19(\mathrm{~s}, 1 \mathrm{H})$, $3.60(\mathrm{~s}, 3 \mathrm{H}), 2.36(\mathrm{~s}, 3 \mathrm{H}), 1.36(\mathrm{~s}, 18 \mathrm{H}) .{ }^{13} \mathrm{C}-\mathrm{NMR}(151 \mathrm{MHz}) \delta 156.98(\mathrm{~s}), 153.95$ (s), 143.67 (s), 136.30 (s), 135.69 (s), 129.86 (s), 129.39 (s), 129.08 (s), 128.82 (s), 127.36 (s), 123.53 (s), 122.54 (s), 120.69 (s), 110.79 (s), $66.98(\mathrm{~s}), 55.56(\mathrm{~s}), 34.30(\mathrm{~s}), 30.17(\mathrm{~s}), 21.46(\mathrm{~s})$.

2,6-Di-tert-butyl-4-((2-chlorophenyl)(tosyl)methyl)phenol (3ad): [15] Pale yellow solid; 75\% yield. ${ }^{1} \mathrm{H}-\mathrm{NMR}$ $\left(600 \mathrm{MHz} \mathrm{CDCl}_{3}\right) \delta 8.35(\mathrm{~d}, J=7.9 \mathrm{~Hz}, 1 \mathrm{H}), 7.46(\mathrm{~d}, J=8.1 \mathrm{~Hz}, 2 \mathrm{H}), 7.37(\mathrm{t}, J=7.6 \mathrm{~Hz}, 1 \mathrm{H}), 7.29(\mathrm{~d}$, $J=8.0 \mathrm{~Hz}, 1 \mathrm{H}), 7.23(\mathrm{t}, J=7.7 \mathrm{~Hz}, 1 \mathrm{H}), 7.16(\mathrm{~s}, 3 \mathrm{H}), 7.15(\mathrm{~s}, 1 \mathrm{H}), 5.88(\mathrm{~s}, 1 \mathrm{H}), 5.23(\mathrm{~s}, 1 \mathrm{H}), 2.37(\mathrm{~s}, 3 \mathrm{H})$, 1.35 (s, 18H). ${ }^{13} \mathrm{C}-\mathrm{NMR}\left(151 \mathrm{MHz}, \mathrm{CDCl}_{3}\right) \delta 153.20$ (s), 143.25 (s), 134.90 (s), 134.72 (s), 133.87 (s), 131.18 (s), 129.10 (s), 128.80 (s), 128.38 (s), 128.17 (s), 128.01 (s), 126.25 (s), 126.05 (s), 121.54 (s), 70.08 (s), 33.28 (s), $29.11(\mathrm{~s}), 20.52(\mathrm{~s})$.

4-((2-Bromophenyl)(tosyl)methyl)-2,6-di-tert-butylphenol (3ae): [15] Pale yellow solid; 82\% yield. ${ }^{1} \mathrm{H}-\mathrm{NMR}$ $\left(600 \mathrm{MHz} \mathrm{CDCl}_{3}\right) \delta 8.35(\mathrm{~d}, J=7.8 \mathrm{~Hz}, 1 \mathrm{H}), 7.47(\mathrm{~d}, J=7.9 \mathrm{~Hz}, 3 \mathrm{H}), 7.41(\mathrm{t}, J=7.6 \mathrm{~Hz}, 1 \mathrm{H}), 7.18(\mathrm{~s}$, 
2H), $7.15(\mathrm{~m}, 3 \mathrm{H}), 5.90(\mathrm{~s}, 1 \mathrm{H}), 5.23(\mathrm{~s}, 1 \mathrm{H}), 2.37(\mathrm{~s}, 3 \mathrm{H}), 1.36(\mathrm{~s}, 18 \mathrm{H}) .{ }^{13} \mathrm{C}-\mathrm{NMR}\left(151 \mathrm{MHz}, \mathrm{CDCl}_{3}\right) \delta$ $154.19(\mathrm{~s}), 144.25$ (s), $135.92(\mathrm{~s}), 135.77(\mathrm{~s}), 133.88$ (s), $133.17(\mathrm{~s}), 130.19$ (s), $129.64(\mathrm{~s}), 129.18$ (s), 129.01 (s), $127.69(\mathrm{~s}), 127.24(\mathrm{~s}), 126.02(\mathrm{~s}), 122.56(\mathrm{~s}), 73.83(\mathrm{~s}), 34.29$ (s), $30.13(\mathrm{~s}), 21.51(\mathrm{~s})$.

2,6-Di-tert-butyl-4-((2-fluorophenyl)(tosyl)methyl)phenol (3af): [15] Pale yellow solid; 74\% yield. ${ }^{1} \mathrm{H}-\mathrm{NMR}$ $\left(600 \mathrm{MHz}, \mathrm{CDCl}_{3}\right) \delta 8.21(\mathrm{t}, J=7.2 \mathrm{~Hz}, 1 \mathrm{H}), 7.46(\mathrm{~d}, J=8.0 \mathrm{~Hz}, 2 \mathrm{H}), 7.29(\mathrm{dd}, J=13.2,6.3 \mathrm{~Hz}, 1 \mathrm{H})$, 7.27-7.22 (m, 1H), $7.16(\mathrm{~s}, 3 \mathrm{H}), 7.15(\mathrm{~s}, 1 \mathrm{H}), 6.96(\mathrm{t}, J=9.1 \mathrm{~Hz}, 1 \mathrm{H}), 5.63(\mathrm{~s}, 1 \mathrm{H}), 5.24(\mathrm{~s}, 1 \mathrm{H}), 2.37(\mathrm{~s}, 3 \mathrm{H})$, $1.35(\mathrm{~s}, 18 \mathrm{H}) .{ }^{13} \mathrm{C}-\mathrm{NMR}\left(151 \mathrm{MHz}, \mathrm{CDCl}_{3}\right) \delta 160.61\left(\mathrm{~d}, J_{\mathrm{C}-F}=247.4 \mathrm{~Hz}\right), 154.22(\mathrm{~s}), 144.23(\mathrm{~s}), 135.96(\mathrm{~s})$, $135.57(\mathrm{~s}), 130.15\left(\mathrm{~d}, J_{\mathrm{C}-F}=1.6 \mathrm{~Hz}\right), 129.97\left(\mathrm{~d}, J_{\mathrm{C}-F}=8.6 \mathrm{~Hz}\right), 129.14(\mathrm{~s}), 129.05(\mathrm{~s}), 127.19(\mathrm{~s}), 124.30(\mathrm{~d}$, $\left.J_{C-F}=3.6 \mathrm{~Hz}\right), 122.73(\mathrm{~s}), 121.57\left(\mathrm{~d}, J_{C-F}=13.5 \mathrm{~Hz}\right), 115.50\left(\mathrm{~d}, J_{C-F}=23.0 \mathrm{~Hz}\right), 67.18\left(\mathrm{~d}, J_{C-F}=4.2 \mathrm{~Hz}\right)$, $34.29(\mathrm{~s}), 30.10(\mathrm{~s}), 21.50(\mathrm{~s})$.

2,6-Di-tert-butyl-4-(tosyl(2-(trifluoromethyl)phenyl)methyl)phenol (3ag): [15] Pale yellow solid; 89\% yield. ${ }^{1} \mathrm{H}-\mathrm{NMR}\left(600 \mathrm{MHz}, \mathrm{CDCl}_{3}\right) \delta 8.61(\mathrm{~d}, J=7.9 \mathrm{~Hz}, 1 \mathrm{H}), 7.68(\mathrm{t}, J=7.7 \mathrm{~Hz}, 1 \mathrm{H}), 7.62(\mathrm{~d}, J=7.8 \mathrm{~Hz}, 1 \mathrm{H})$, $7.44(\mathrm{~m}, 3 \mathrm{H}), 7.15(\mathrm{~m}, 4 \mathrm{H}), 5.59(\mathrm{~s}, 1 \mathrm{H}), 5.23(\mathrm{~s}, 1 \mathrm{H}), 2.36(\mathrm{~s}, 3 \mathrm{H}), 1.34(\mathrm{~s}, 18 \mathrm{H}) .{ }^{13} \mathrm{C}-\mathrm{NMR}(151 \mathrm{MHz}$, $\left.\mathrm{CDCl}_{3}\right) \delta 154.20(\mathrm{~s}), 144.34(\mathrm{~s}), 135.87(\mathrm{~s}), 135.59(\mathrm{~s}), 133.00(\mathrm{~s}), 131.99(\mathrm{~s}), 130.47(\mathrm{~s}), 129.18(\mathrm{~s}), 129.10(\mathrm{~s})$, $\delta 129.64-128.96(\mathrm{~m}), 128.25(\mathrm{~s}), 127.10(\mathrm{~s}), 126.45\left(\mathrm{q}, J_{C-F}=5.9 \mathrm{~Hz}\right), 124.12\left(\mathrm{q}, J_{C-F}=274.4 \mathrm{~Hz}\right) .122 .73(\mathrm{~s})$, $71.00(\mathrm{~s}), 34.29(\mathrm{~s}), 30.10(\mathrm{~s}), 21.48(\mathrm{~s})$.

2,6-Di-tert-butyl-4-(m-tolyl(tosyl)methyl)phenol (3ah): [15] Pale yellow solid; 84\% yield. ${ }^{1} \mathrm{H}$-NMR $\left(600 \mathrm{MHz}, \mathrm{CDCl}_{3}\right) \delta 7.46(\mathrm{~d}, J=7.5 \mathrm{~Hz}, 1 \mathrm{H}), 7.42(\mathrm{~d}, J=7.7 \mathrm{~Hz}, 2 \mathrm{H}), 7.39(\mathrm{~s}, 1 \mathrm{H}), 7.28-7.19(\mathrm{~m}, 1 \mathrm{H})$, $7.13(\mathrm{~m}, 5 \mathrm{H}), 5.20(\mathrm{~s}, 1 \mathrm{H}), 5.12(\mathrm{~s}, 1 \mathrm{H}), 2.35(\mathrm{~d}, J=9.0 \mathrm{~Hz}, 3 \mathrm{H}), 2.33(\mathrm{~s}, 3 \mathrm{H}), 1.35(\mathrm{~s}, 18 \mathrm{H}) ;{ }^{13} \mathrm{C}-\mathrm{NMR}$ $\left(151 \mathrm{MHz}, \mathrm{CDCl}_{3}\right) \delta 154.03(\mathrm{~s}), 143.92(\mathrm{~s}), 138.21(\mathrm{~s}), 135.84(\mathrm{~s}), 135.81(\mathrm{~s}), 133.43(\mathrm{~s}), 130.86(\mathrm{~s}), 129.12(\mathrm{~s})$, 128.99 (s), 128.49 (s), 127.10 (s), 126.88 (s), 123.65 (s), 76.92 (s), 34.28 (s), 30.12 (s), 21.46 (s).

2,6-Di-tert-butyl-4-((3-methoxyphenyl)(tosyl)methyl)phenol (3ai): [15] Pale yellow solid; 81\% yield. ${ }^{1} \mathrm{H}-\mathrm{NMR}\left(600 \mathrm{MHz}, \mathrm{CDCl}_{3}\right) \delta 7.43(\mathrm{~d}, J=7.8 \mathrm{~Hz}, 2 \mathrm{H}), 7.27-7.23(\mathrm{~m}, 1 \mathrm{H}), 7.19(\mathrm{~d}, J=5.4 \mathrm{~Hz}$, $2 \mathrm{H}), 7.17-7.11(\mathrm{~m}, 4 \mathrm{H}), 6.86(\mathrm{~d}, J=8.0 \mathrm{~Hz}, 1 \mathrm{H}), 5.21(\mathrm{~s}, 1 \mathrm{H}), 5.13(\mathrm{~s}, 1 \mathrm{H}), 3.79(\mathrm{~s}, 3 \mathrm{H}), 2.36(\mathrm{~s}, 3 \mathrm{H}), 1.35(\mathrm{~s}$, 18H). ${ }^{13} \mathrm{C}-\mathrm{NMR}\left(151 \mathrm{MHz}, \mathrm{CDCl}_{3}\right) \delta 159.65$ (s), 154.08 (s), 143.99 (s), 135.87 (s), 135.74 (s), 134.93 (s), $129.53(\mathrm{~s}), 129.11(\mathrm{~s}), 129.03(\mathrm{~s}), 127.07(\mathrm{~s}), 123.45(\mathrm{~s}), 122.39(\mathrm{~s}), 115.33(\mathrm{~s}), 114.42(\mathrm{~s}), 76.78(\mathrm{~s}), 55.22(\mathrm{~s})$, $34.29(\mathrm{~s}), 30.13(\mathrm{~s}), 21.47(\mathrm{~s})$.

4-((3-Bromophenyl)(tosyl)methyl)-2,6-di-tert-butylphenol (3aj): [15] Pale yellow solid; 77\% yield. ${ }^{1} \mathrm{H}-\mathrm{NMR}$ $\left(600 \mathrm{MHz}, \mathrm{CDCl}_{3}\right) \delta 7.69(\mathrm{~s}, 1 \mathrm{H}), 7.66(\mathrm{~d}, J=7.8 \mathrm{~Hz}, 1 \mathrm{H}), 7.45(\mathrm{~d}, J=8.0 \mathrm{~Hz}, 1 \mathrm{H}), 7.42(\mathrm{~d}, J=8.1 \mathrm{~Hz}, 2 \mathrm{H})$, $7.23(\mathrm{t}, J=7.9 \mathrm{~Hz}, 1 \mathrm{H}), 7.15(\mathrm{~d}, J=8.0 \mathrm{~Hz}, 2 \mathrm{H}), 7.10(\mathrm{~s}, 2 \mathrm{H}), 5.24(\mathrm{~s}, 1 \mathrm{H}), 5.11(\mathrm{~s}, 1 \mathrm{H}), 2.37(\mathrm{~s}, 3 \mathrm{H}), 1.35(\mathrm{~s}$, 18H). ${ }^{13} \mathrm{C}-\mathrm{NMR}\left(151 \mathrm{MHz}, \mathrm{CDCl}_{3}\right) \delta 154.24$ (s), 144.32 (s), 136.08 (s), 135.82 (s), 135.35 (s), 133.15 (s), 131.46 (s), 130.07 (s), 129.16 (s), 129.10 (s), 128.34 (s), 127.02 (s), 122.90 (s), 122.55 (s), 76.15 (s), 34.31 (s), $30.10(\mathrm{~s}), 21.49(\mathrm{~s})$.

2,6-Di-tert-butyl-4-((3-fluorophenyl)(tosyl)methyl)phenol (3ak): [15] Pale yellow solid; 78\% yield. ${ }^{1} \mathrm{H}-\mathrm{NMR}$ $\left(400 \mathrm{MHz}, \mathrm{CDCl}_{3}\right) \delta 7.43(\mathrm{~d}, J=8.2 \mathrm{~Hz}, 2 \mathrm{H}), 7.41-7.36(\mathrm{~m}, 2 \mathrm{H}), 7.31(\mathrm{td}, J=8.0,6.0 \mathrm{~Hz}, 1 \mathrm{H}), 7.15(\mathrm{~d}$, $J=8.0 \mathrm{~Hz}, 2 \mathrm{H}), 7.11(\mathrm{~s}, 2 \mathrm{H}), 7.02(\mathrm{tdd}, J=8.4,2.5,0.8 \mathrm{~Hz}, 1 \mathrm{H}), 5.25(\mathrm{~s}, 1 \mathrm{H}), 5.15(\mathrm{~s}, 1 \mathrm{H}), 2.37(\mathrm{~s}, 3 \mathrm{H})$, $1.35(\mathrm{~s}, 18 \mathrm{H}) .{ }^{13} \mathrm{C}-\mathrm{NMR}\left(151 \mathrm{MHz}, \mathrm{CDCl}_{3}\right) \delta 162.67\left(\mathrm{~d}, J_{\mathrm{C}-F}=246.5 \mathrm{~Hz}\right), 154.23(\mathrm{~s}), 144.24(\mathrm{~s}), 136.06(\mathrm{~s})$, $135.91\left(\mathrm{~d}, J_{\mathrm{C}-F}=7.3 \mathrm{~Hz}\right), 135.46(\mathrm{~s}), 130.02\left(\mathrm{~d}, J_{\mathrm{C}-F}=8.3 \mathrm{~Hz}\right), 129.13(\mathrm{~s}), 129.08(\mathrm{~s}), 127.01(\mathrm{~s}), 125.71(\mathrm{~d}$, $\left.J_{C-F}=2.8 \mathrm{~Hz}\right), 123.04(\mathrm{~s}), 117.05\left(\mathrm{~d}, J_{C-F}=23.0 \mathrm{~Hz}\right), 115.35\left(\mathrm{~d}, J_{C-F}=21.1 \mathrm{~Hz}\right), 76.22(\mathrm{~s}), 34.29(\mathrm{~s}), 30.10$ (s), $21.43(\mathrm{~s})$.

2,6-Di-tert-butyl-4-(tosyl(3-(trifluoromethyl)phenyl)methyl)phenol (3al): [15] Pale yellow solid; 60\% yield. ${ }^{1} \mathrm{H}-\mathrm{NMR}\left(600 \mathrm{MHz}, \mathrm{CDCl}_{3}\right) \delta 7.91(\mathrm{~d}, J=7.7 \mathrm{~Hz}, 1 \mathrm{H}), 7.81(\mathrm{~s}, 1 \mathrm{H}), 7.58(\mathrm{~d}, J=7.7 \mathrm{~Hz}, 1 \mathrm{H}), 7.49(\mathrm{t}$, $J=7.8 \mathrm{~Hz}, 1 \mathrm{H}), 7.42(\mathrm{~d}, J=8.0 \mathrm{~Hz}, 2 \mathrm{H}), 7.15(\mathrm{~s}, 1 \mathrm{H}), 7.13(\mathrm{~s}, 3 \mathrm{H}), 5.27(\mathrm{~s}, 1 \mathrm{H}), 5.23(\mathrm{~s}, 1 \mathrm{H}), 2.36(\mathrm{~s}, 3 \mathrm{H})$, $1.35(\mathrm{~s}, 18 \mathrm{H}) .{ }^{13} \mathrm{C}-\mathrm{NMR}\left(151 \mathrm{MHz}, \mathrm{CDCl}_{3}\right) \delta 154.33(\mathrm{~s}), 144.47(\mathrm{~s}), 136.20(\mathrm{~s}), 135.18(\mathrm{~s}), 134.71(\mathrm{~s}), 133.22$ $(\mathrm{s}), 130.94\left(\mathrm{q}, J_{\mathrm{C}-F}=32.5 \mathrm{~Hz}\right), 129.20(\mathrm{~s}), 129.11\left(\mathrm{~d}, J_{\mathrm{C}-F}=3.5 \mathrm{~Hz}\right), 127.20-126.83(\mathrm{~m}), 125.29-125.02(\mathrm{~m})$, $123.90\left(\mathrm{q}, J_{C-F}=272.4 \mathrm{~Hz}\right) \cdot 122.70(\mathrm{~s}), 76.29(\mathrm{~s}), 34.32(\mathrm{~s}), 30.08(\mathrm{~s}), 21.45(\mathrm{~s})$. 
2,6-Di-tert-butyl-4-(p-tolyl(tosyl)methyl)phenol (3am): [15] Pale yellow solid; 80\% yield. ${ }^{1} \mathrm{H}-\mathrm{NMR}$ $\left(600 \mathrm{MHz}, \mathrm{CDCl}_{3}\right) \delta 7.50(\mathrm{~d}, J=8.0 \mathrm{~Hz}, 2 \mathrm{H}), 7.42(\mathrm{~d}, J=8.1 \mathrm{~Hz}, 2 \mathrm{H}), 7.15(\mathrm{~d}, J=8.2 \mathrm{~Hz}, 4 \mathrm{H}), 7.12(\mathrm{~d}$, $J=8.1 \mathrm{~Hz}, 2 \mathrm{H}), 5.19(\mathrm{~s}, 1 \mathrm{H}), 5.13(\mathrm{~s}, 1 \mathrm{H}), 2.36(\mathrm{~s}, 3 \mathrm{H}), 2.33(\mathrm{~s}, 3 \mathrm{H}), 1.35(\mathrm{~s}, 18 \mathrm{H}) .{ }^{13} \mathrm{C}-\mathrm{NMR}(151 \mathrm{MHz}$, $\left.\mathrm{CDCl}_{3}\right) \delta 153.99(\mathrm{~s}), 143.87(\mathrm{~s}), 138.20(\mathrm{~s}), 135.84(\mathrm{~s}), 130.49(\mathrm{~s}), 129.84(\mathrm{~s}), 129.33(\mathrm{~s}), 129.09(\mathrm{~s}), 128.99$ (s), $127.03(\mathrm{~s}), 123.75(\mathrm{~s}), 76.67(\mathrm{~s}), 34.28(\mathrm{~s}), 30.13(\mathrm{~s}), 21.47(\mathrm{~s}), 21.09(\mathrm{~s})$.

2,6-Di-tert-butyl-4-((4-methoxyphenyl)(tosyl)methyl)phenol (3an): [15] Pale yellow solid; 79\% yield. ${ }^{1} \mathrm{H}-\mathrm{NMR}\left(600 \mathrm{MHz}, \mathrm{CDCl}_{3}\right) \delta 7.53(\mathrm{~d}, J=8.6 \mathrm{~Hz}, 2 \mathrm{H}), 7.42(\mathrm{~d}, J=8.0 \mathrm{~Hz}, 2 \mathrm{H}), 7.15(\mathrm{~s}, 2 \mathrm{H}), 7.12(\mathrm{~d}$, $J=8.0 \mathrm{~Hz}, 2 \mathrm{H}), 6.87(\mathrm{~d}, J=8.6 \mathrm{~Hz}, 2 \mathrm{H}), 5.20(\mathrm{~s}, 1 \mathrm{H}), 5.13(\mathrm{~s}, 1 \mathrm{H}), 3.79(\mathrm{~s}, 3 \mathrm{H}), 2.35(\mathrm{~s}, 3 \mathrm{H}), 1.35(\mathrm{~s}, 18 \mathrm{H})$. ${ }^{13} \mathrm{C}-\mathrm{NMR}\left(151 \mathrm{MHz}, \mathrm{CDCl}_{3}\right) \delta 155.77$ (s), 150.04 (s), 139.94 (s), 131.93 (s), 131.87 (s), 127.28 (s), 125.13 (s), $125.08(\mathrm{~s}), 123.06(\mathrm{~s}), 121.53(\mathrm{~s}), 119.87(\mathrm{~s}), 110.15(\mathrm{~s}), 72.34(\mathrm{~s}), 51.33(\mathrm{~s}), 30.35(\mathrm{~s}), 27.78-23.72(\mathrm{~m})$, $17.53(\mathrm{~s})$.

2,6-Di-tert-butyl-4-((4-chlorophenyl)(tosyl)methyl)phenol (3ao): [15] Pale yellow solid; 81\% yield. ${ }^{1} \mathrm{H}-\mathrm{NMR}$ $\left(600 \mathrm{MHz}, \mathrm{CDCl}_{3}\right) \delta 7.57(\mathrm{~d}, J=8.4 \mathrm{~Hz}, 2 \mathrm{H}), 7.42(\mathrm{~d}, J=8.1 \mathrm{~Hz}, 2 \mathrm{H}), 7.32(\mathrm{~d}, J=8.4 \mathrm{~Hz}, 2 \mathrm{H}), 7.14(\mathrm{~d}$, $J=8.1 \mathrm{~Hz}, 2 \mathrm{H}), 7.09(\mathrm{~s}, 2 \mathrm{H}), 5.23(\mathrm{~s}, 1 \mathrm{H}), 5.14(\mathrm{~s}, 1 \mathrm{H}), 2.37(\mathrm{~s}, 3 \mathrm{H}), 1.35(\mathrm{~s}, 18 \mathrm{H}) .{ }^{13} \mathrm{C}-\mathrm{NMR}(151 \mathrm{MHz}$, $\left.\mathrm{CDCl}_{3}\right) \delta 154.18(\mathrm{~s}), 144.23(\mathrm{~s}), 136.05(\mathrm{~s}), 135.45(\mathrm{~s}), 134.54(\mathrm{~s}), 132.16(\mathrm{~s}), 131.25(\mathrm{~s}), 129.15(\mathrm{~s}), 129.07(\mathrm{~s})$, $128.82(\mathrm{~s}), 126.96$ (s), 123.10 (s), 76.05 (s), 34.30 (s), 30.10 (s), 21.50 (s).

4-((4-Bromophenyl)(tosyl)methyl)-2,6-di-tert-butylphenol (3ap): [15] Pale yellow solid; 79\% yield. ${ }^{1} \mathrm{H}-\mathrm{NMR}$ $\left(400 \mathrm{MHz}, \mathrm{CDCl}_{3}\right) \delta 7.54-7.45(\mathrm{~m}, 4 \mathrm{H}), 7.42(\mathrm{~d}, J=8.2 \mathrm{~Hz}, 2 \mathrm{H}), 7.15(\mathrm{~d}, J=8.1 \mathrm{~Hz}, 2 \mathrm{H}), 7.09(\mathrm{~s}, 2 \mathrm{H})$, $5.24(\mathrm{~s}, 1 \mathrm{H}), 5.13(\mathrm{~s}, 1 \mathrm{H}), 2.37(\mathrm{~s}, 3 \mathrm{H}), 1.34(\mathrm{~s}, 18 \mathrm{H}) .{ }^{13} \mathrm{C}-\mathrm{NMR}\left(101 \mathrm{MHz}, \mathrm{CDCl}_{3}\right) \delta 154.20(\mathrm{~s}), 144.28(\mathrm{~s})$, $136.02(\mathrm{~s}), 135.35(\mathrm{~s}), 132.67(\mathrm{~s}), 131.79(\mathrm{~s}), 131.54(\mathrm{~s}), 129.17(\mathrm{~s}), 129.06(\mathrm{~s}), 126.95(\mathrm{~s}), 122.98(\mathrm{~s}), 122.76(\mathrm{~s})$, $76.08(\mathrm{~s}), 34.30(\mathrm{~s}), 30.10(\mathrm{~s}), 21.53(\mathrm{~s})$.

2,6-Di-tert-butyl-4-((4-fluorophenyl)(tosyl)methyl)phenol (3aq): [15] Pale yellow solid; 70\% yield. ${ }^{1} \mathrm{H}-\mathrm{NMR}$ $\left(600 \mathrm{MHz} \mathrm{CDCl}_{3}\right) \delta 7.60(\mathrm{dd}, J=8.2,5.4 \mathrm{~Hz}, 2 \mathrm{H}), 7.42(\mathrm{~d}, J=8.0 \mathrm{~Hz}, 2 \mathrm{H}), 7.14(\mathrm{~d}, J=8.0 \mathrm{~Hz}, 2 \mathrm{H}), 7.12$ $(\mathrm{s}, 2 \mathrm{H}), 7.04(\mathrm{t}, J=8.5 \mathrm{~Hz}, 2 \mathrm{H}), 5.23(\mathrm{~s}, 1 \mathrm{H}), 5.16(\mathrm{~s}, 1 \mathrm{H}), 2.36(\mathrm{~s}, 3 \mathrm{H}), 1.35(\mathrm{~s}, 18 \mathrm{H}) \cdot{ }^{13} \mathrm{C}-\mathrm{NMR}(151 \mathrm{MHz}$, $\left.\mathrm{CDCl}_{3}\right) \delta 162.79\left(\mathrm{~d}, J_{\mathrm{C}-F}=248.1 \mathrm{~Hz}\right), 154.13(\mathrm{~s}), 144.15(\mathrm{~s}), 136.03(\mathrm{~s}), 135.54(\mathrm{~s}), 131.73\left(\mathrm{~d}, J_{\mathrm{C}-F}=8.2 \mathrm{~Hz}\right)$, $129.44\left(\mathrm{~d}, J_{\mathrm{C}-F}=3.2 \mathrm{~Hz}\right), 129.11(\mathrm{~s}), 129.06(\mathrm{~s}), 126.97(\mathrm{~s}), 123.33(\mathrm{~s}), 115.57\left(\mathrm{~d}, J_{\mathrm{C}-F}=21.5 \mathrm{~Hz}\right), 75.98(\mathrm{~s})$, $34.30(\mathrm{~s}), 30.11(\mathrm{~s}), 21.47(\mathrm{~s})$.

2,6-Di-tert-butyl-4-(tosyl(4-(trifluoromethyl)phenyl)methyl)phenol (3ar): [15] Pale yellow solid; 77\% yield. ${ }^{1} \mathrm{H}-\mathrm{NMR}\left(600 \mathrm{MHz}, \mathrm{CDCl}_{3}\right) \delta 7.78(\mathrm{~d}, J=8.1 \mathrm{~Hz}, 2 \mathrm{H}), 7.62(\mathrm{~d}, J=8.2 \mathrm{~Hz}, 2 \mathrm{H}), 7.42(\mathrm{~d}, J=8.1 \mathrm{~Hz}, 2 \mathrm{H})$, $7.15(\mathrm{~d}, J=8.0 \mathrm{~Hz}, 2 \mathrm{H}), 7.09(\mathrm{~s}, 2 \mathrm{H}), 5.25(\mathrm{~s}, 1 \mathrm{H}), 5.22(\mathrm{~s}, 1 \mathrm{H}), 2.37(\mathrm{~s}, 3 \mathrm{H}), 1.35(\mathrm{~s}, 18 \mathrm{H}) .{ }^{13} \mathrm{C}-\mathrm{NMR}$ $\left(151 \mathrm{MHz}, \mathrm{CDCl}_{3}\right) \delta 154.29(\mathrm{~s}), 144.41(\mathrm{~s}), 137.69(\mathrm{~s}), 136.17(\mathrm{~s}), 135.29(\mathrm{~s}), 130.55\left(\mathrm{q}, J_{\mathrm{C}-F}=32.6 \mathrm{~Hz}\right)$, $130.28(\mathrm{~s}), 129.18(\mathrm{~s}), 129.07(\mathrm{~s}), 126.97(\mathrm{~s}), 125.53\left(\mathrm{q}, J_{\mathrm{C}-F}=3.6 \mathrm{~Hz}\right), 123.96\left(\mathrm{q}, J_{C-F}=272.1 \mathrm{~Hz}\right) .122 .83(\mathrm{~s})$, $76.32(\mathrm{~s}), 34.30(\mathrm{~s}), 30.08(\mathrm{~s}), 21.49(\mathrm{~s})$.

2,6-Di-tert-butyl-4-((4-nitrophenyl)(tosyl)methyl)phenol (3as): [15] Pale yellow solid; 72\% yield. ${ }^{1} \mathrm{H}-\mathrm{NMR}$ $\left(400 \mathrm{MHz}, \mathrm{CDCl}_{3}\right) \delta 8.22(\mathrm{~d}, J=8.9 \mathrm{~Hz}, 2 \mathrm{H}), 7.85(\mathrm{~d}, J=8.8 \mathrm{~Hz}, 2 \mathrm{H}), 7.43(\mathrm{~d}, J=8.3 \mathrm{~Hz}, 2 \mathrm{H}), 7.17(\mathrm{~d}$, $J=8.0 \mathrm{~Hz}, 2 \mathrm{H}), 7.07(\mathrm{~s}, 2 \mathrm{H}), 5.29(\mathrm{~s}, 1 \mathrm{H}), 5.27(\mathrm{~s}, 1 \mathrm{H}), 2.38(\mathrm{~s}, 3 \mathrm{H}), 1.34(\mathrm{~s}, 18 \mathrm{H}) .{ }^{13} \mathrm{C}-\mathrm{NMR}(151 \mathrm{MHz}$, $\left.\mathrm{CDCl}_{3}\right) \delta 153.76(\mathrm{~s}), 148.51(\mathrm{~d}, J=2.9 \mathrm{~Hz}), 147.13(\mathrm{~s}), 143.63(\mathrm{~s}), 137.22(\mathrm{~s}), 136.63(\mathrm{~s}), 129.98(\mathrm{~s}), 129.55$ (s), $128.21(\mathrm{~s}), 127.26(\mathrm{~s}), 124.18(\mathrm{~s}), 123.50$ (s), 61.32 (s), 34.31 (s), 30.04 (s), 21.45 (s).

2,6-Di-tert-butyl-4-((2,4-dichlorophenyl)(tosyl)methyl)phenol (3at): [15] Pale yellow solid; 68\% yield. ${ }^{1} \mathrm{H}-\mathrm{NMR}\left(600 \mathrm{MHz}, \mathrm{CDCl}_{3}\right) \delta 8.29(\mathrm{~d}, J=8.5 \mathrm{~Hz}, 1 \mathrm{H}), 7.46(\mathrm{~d}, J=8.0 \mathrm{~Hz}, 2 \mathrm{H}), 7.37(\mathrm{~d}, J=8.5 \mathrm{~Hz}, 1 \mathrm{H})$, $7.33(\mathrm{~s}, 1 \mathrm{H}), 7.18(\mathrm{~d}, J=7.9 \mathrm{~Hz}, 2 \mathrm{H}), 7.10(\mathrm{~s}, 2 \mathrm{H}), 5.79(\mathrm{~s}, 1 \mathrm{H}), 5.25(\mathrm{~s}, 1 \mathrm{H}), 2.39(\mathrm{~s}, 1 \mathrm{H}), 1.35(\mathrm{~s}, 18 \mathrm{H})$. ${ }^{13} \mathrm{C}-\mathrm{NMR}\left(151 \mathrm{MHz}, \mathrm{CDCl}_{3}\right) \delta 154.31$ (s), 144.50 (s), 136.06 (s), 135.56 (s), 135.47 (s), 134.79 (s), 130.90 (s), 130.83 (s), 129.62 (s), 129.29 (s), 128.99 (s), 127.44 (s), 127.08 (s), 122.18 (s), 70.63 (s), 34.29 (s), 30.09 (s), $21.53(\mathrm{~s})$.

2,6-Di-tert-butyl-4-((1,3-dihydroisobenzofuran-4-yl)(tosyl)methyl)phenol (3au): [15] Pale yellow solid; 68\% yield. ${ }^{1} \mathrm{H}-\mathrm{NMR}\left(600 \mathrm{MHz}, \mathrm{CDCl}_{3}\right) \delta 7.43(\mathrm{~d}, J=8.1 \mathrm{~Hz}, 1 \mathrm{H}), 7.20(\mathrm{~s}, 1 \mathrm{H}), 7.13(\mathrm{~d}, J=9.6 \mathrm{~Hz}, 1 \mathrm{H}), 7.01$ $(\mathrm{d}, J=8.1 \mathrm{~Hz}, 1 \mathrm{H}), 6.76(\mathrm{~d}, J=8.1 \mathrm{~Hz}, 1 \mathrm{H}), 5.95(\mathrm{~d}, J=3.4 \mathrm{~Hz}, 1 \mathrm{H}), 5.21(\mathrm{~s}, 1 \mathrm{H}), 5.08(\mathrm{~s}, 1 \mathrm{H}), 2.36(\mathrm{~s}$, 
1H), 1.35 (s, 1H). ${ }^{13} \mathrm{C}-\mathrm{NMR}\left(151 \mathrm{MHz}, \mathrm{CDCl}_{3}\right) \delta 154.19$ (s), 148.02 (s), 147.93 (s), 144.14 (s), 136.09 (s), 135.86 (s), 129.20 (s), 127.14 (s), 127.03 (s), 124.14 (s), 123.83 (s), 110.36 (s), 108.45 (s), 101.37 (s), 76.60 (s), 34.43 (s), 32.20-27.96 (m), 21.62 (s); HRMS calculated for $[\mathrm{M}+\mathrm{Na}]^{+} \mathrm{C}_{29} \mathrm{H}_{34} \mathrm{O}_{5} \mathrm{SNa}^{+}, \mathrm{m} / \mathrm{z}$ 517.2019, found 517.2020.

2,6-Di-tert-butyl-4-(naphthalen-1-yl(tosyl)methyl)phenol (3av): [15] Pale yellow solid; 78\% yield. ${ }^{1} \mathrm{H}-\mathrm{NMR}$ $\left(600 \mathrm{MHz}, \mathrm{CDCl}_{3}\right) \delta 8.55(\mathrm{~d}, J=7.2 \mathrm{~Hz}, 1 \mathrm{H}), 7.86(\mathrm{~d}, J=7.8 \mathrm{~Hz}, 1 \mathrm{H}), 7.82(\mathrm{t}, J=7.8 \mathrm{~Hz}, 2 \mathrm{H}), 7.59(\mathrm{t}$, $J=7.7 \mathrm{~Hz}, 1 \mathrm{H}), 7.46(\mathrm{~d}, J=7.9 \mathrm{~Hz}, 2 \mathrm{H}), 7.45-7.39(\mathrm{~m}, 2 \mathrm{H}), 7.25(\mathrm{~s}, 1 \mathrm{H}), 7.14(\mathrm{~s}, 1 \mathrm{H}), 7.12(\mathrm{~d}, J=7.9 \mathrm{~Hz}$, 2H), $6.05(\mathrm{~s}, 1 \mathrm{H}), 5.19(\mathrm{~s}, 1 \mathrm{H}), 2.33(\mathrm{~s}, 3 \mathrm{H}), 1.31(\mathrm{~s}, 18 \mathrm{H}) .{ }^{13} \mathrm{C}-\mathrm{NMR}\left(151 \mathrm{MHz}, \mathrm{CDCl}_{3}\right) \delta 154.12(\mathrm{~s}), 144.09$ $(\mathrm{s}), 136.00(\mathrm{~s}), 135.77(\mathrm{~s}), 134.12(\mathrm{~s}), 131.79(\mathrm{~s}), 129.79(\mathrm{~s}), 129.16(\mathrm{~s}), 129.13(\mathrm{~s}), 128.92(\mathrm{~s}), 127.32(\mathrm{~s}), 126.85$ (s), 126.50 (s), 125.49 (s), 125.32 (s), 123.36 (s), 122.37 (s), 71.18 (s), 34.24 (s), 30.08 (s), 21.46 (s).

2,6-Dimethyl-4-(phenyl(tosyl)methyl)phenol (3aw): [15] Pale yellow solid; 63\% yield. ${ }^{1} \mathrm{H}-\mathrm{NMR}(600 \mathrm{MHz}$, $\left.\mathrm{CDCl}_{3}\right) \delta$ 7.54-7.44 (m, 4H), $7.28(\mathrm{~m}, 3 \mathrm{H}), 7.15(\mathrm{~m}, 4 \mathrm{H}), 5.13(\mathrm{~s}, 1 \mathrm{H}), 4.70(\mathrm{~s}, 1 \mathrm{H}), 2.37(\mathrm{~s}, 3 \mathrm{H}), 2.20(\mathrm{~s}, 6 \mathrm{H})$. ${ }^{13} \mathrm{C}-\mathrm{NMR}\left(151 \mathrm{MHz}, \mathrm{CDCl}_{3}\right) \delta 152.62(\mathrm{~s}), 144.22(\mathrm{~s}), 135.65$ (s), 133.77 (s), 130.25 (s), $129.86(\mathrm{~s}), 129.15$ (s), $129.08(\mathrm{~s}), 128.56(\mathrm{~s}), 128.35$ (s), $124.34(\mathrm{~s}), 123.28(\mathrm{~s}), 76.09$ (s), 21.53 (s), 15.90 (s).

2,6-Diisopropyl-4-(phenyl(tosyl)methyl)phenol (3ax): [15] Pale yellow solid; 79\% yield. ${ }^{1} \mathrm{H}-\mathrm{NMR}(600 \mathrm{MHz}$, $\left.\mathrm{CDCl}_{3}\right) \delta 7.59(\mathrm{~d}, J=7.8 \mathrm{~Hz}, 1 \mathrm{H}), 7.45(\mathrm{~d}, J=7.4 \mathrm{~Hz}, 1 \mathrm{H}), 7.37-7.28(\mathrm{~m}, 1 \mathrm{H}), 7.13(\mathrm{~d}, J=8.0 \mathrm{~Hz}, 1 \mathrm{H})$, $7.09(\mathrm{~s}, 1 \mathrm{H}), 5.20(\mathrm{~s}, 1 \mathrm{H}), 4.81(\mathrm{~s}, 1 \mathrm{H}), 3.19-2.90(\mathrm{~m}, 1 \mathrm{H}), 2.35(\mathrm{~s}, 1 \mathrm{H}), 1.22(\mathrm{~d}, J=6.9 \mathrm{~Hz}, 1 \mathrm{H}), 1.14(\mathrm{~d}$, $J=6.8 \mathrm{~Hz}, 1 \mathrm{H}) .{ }^{13} \mathrm{C}-\mathrm{NMR}\left(151 \mathrm{MHz}, \mathrm{CDCl}_{3}\right) \delta 150.23(\mathrm{~s}), 144.02(\mathrm{~s}), 133.77(\mathrm{~s}), 133.48(\mathrm{~s}), 129.99(\mathrm{~s})$, 129.08 (s), 129.05 (s), 128.58 (s), 128.36 (s), 125.59 (s), 124.87 (s), 99.99 (s), 76.62 (s), 27.21 (s), 22.61 (s), $22.53(\mathrm{~s}), 21.48(\mathrm{~s})$.

2,6-Di-tert-butyl-4-(1-tosylethyl)phenol (3ay): [15] Pale yellow solid; $80 \%$ yield. ${ }^{1} \mathrm{H}-\mathrm{NMR}(600 \mathrm{MHz}$,) $\delta$ $7.33(\mathrm{~d}, J=8.2 \mathrm{~Hz}, 2 \mathrm{H}), 7.15(\mathrm{~d}, J=8.2 \mathrm{~Hz}, 2 \mathrm{H}), 6.78(\mathrm{~s}, 2 \mathrm{H}), 5.21(\mathrm{~s}, 1 \mathrm{H}), 4.13(\mathrm{q}, J=7.2 \mathrm{~Hz}, 1 \mathrm{H}), 2.38$ $(\mathrm{s}, 3 \mathrm{H}), 1.77(\mathrm{~d}, J=7.2 \mathrm{~Hz}, 3 \mathrm{H}), 1.32(\mathrm{~s}, 18 \mathrm{H}) .{ }^{13} \mathrm{C}-\mathrm{NMR}(151 \mathrm{MHz},) \delta 154.13(\mathrm{~s}), 143.98$ (s), 135.75 (s), $134.32(\mathrm{~s}), 129.31$ (s), 129.04 (s), 126.21 (s), 124.39 (s), 66.53 (s), 34.23 (s), 30.13 (s), 21.50 (s), 13.45 (s).

2,6-Di-tert-butyl-4-(phenyl(phenylsulfonyl)methyl)phenol (4aa): [16] Pale yellow solid; 92\% yield. ${ }^{1} \mathrm{H}-\mathrm{NMR}$ $\left(600 \mathrm{MHz}, \mathrm{CDCl}_{3}\right) \delta 7.62(\mathrm{~d}, J=7.2 \mathrm{~Hz}, 2 \mathrm{H}), 7.57(\mathrm{~d}, J=7.8 \mathrm{~Hz}, 2 \mathrm{H}), 7.50(\mathrm{t}, J=7.3 \mathrm{~Hz}, 1 \mathrm{H}), 7.34(\mathrm{dd}$, $J=15.7,8.5 \mathrm{~Hz}, 5 \mathrm{H}), 7.19(\mathrm{~s}, 2 \mathrm{H}), 5.24(\mathrm{~s}, 1 \mathrm{H}), 5.21(\mathrm{~s}, 1 \mathrm{H}), 1.36(\mathrm{~s}, 18 \mathrm{H}) \cdot{ }^{13} \mathrm{C}-\mathrm{NMR}\left(151 \mathrm{MHz}, \mathrm{CDCl}_{3}\right) \delta$ $154.15(\mathrm{~s}), 138.65$ (s), 135.97 (s), 133.45 (s), 133.13 (s), 130.00 (s), 129.08 (s), 128.67 (s), 128.47 (s), 128.44 (s), $127.10(\mathrm{~s}), 123.29(\mathrm{~s}), 34.34(\mathrm{~s}), 30.18(\mathrm{~s})$.

2,6-Di-tert-butyl-4-(((4-(tert-butyl)phenyl)sulfonyl)(phenyl)methyl)phenol (4ab): [16] Pale yellow solid; 77\% yield. ${ }^{1} \mathrm{H}-\mathrm{NMR}\left(600 \mathrm{MHz}, \mathrm{CDCl}_{3}\right) \delta 7.63(\mathrm{~d}, J=7.1 \mathrm{~Hz}, 2 \mathrm{H}), 7.45(\mathrm{~d}, J=8.4 \mathrm{~Hz}, 2 \mathrm{H}), 7.38-7.30(\mathrm{~m}, 5 \mathrm{H})$, $7.12(\mathrm{~s}, 2 \mathrm{H}), 5.21(\mathrm{~s}, 1 \mathrm{H}), 5.18(\mathrm{~s}, 1 \mathrm{H}), 1.34(\mathrm{~s}, 18 \mathrm{H}), 1.29(\mathrm{~s}, 9 \mathrm{H}) .{ }^{13} \mathrm{C}-\mathrm{NMR}\left(151 \mathrm{MHz}, \mathrm{CDCl}_{3}\right) \delta 157.07(\mathrm{~s})$, $154.04(\mathrm{~s}), 135.85$ (s), 135.59 (s), $133.66(\mathrm{~s}), 129.96$ (s), 129.01 (s), 128.60 (s), 128.33 (s), 127.13 (s), 125.42 (s), $123.57(\mathrm{~s}), 76.88(\mathrm{~s}), 35.12(\mathrm{~s}), 34.29(\mathrm{~s}), 31.04(\mathrm{~s}), 30.16(\mathrm{~s})$.

2,6-Di-tert-butyl-4-((4-methoxyphenyl)sulfonyl)(phenyl)methyl)phenol (4ac): [16] Pale yellow solid; 96\% yield. ${ }^{1} \mathrm{H}-\mathrm{NMR}\left(400 \mathrm{MHz}, \mathrm{CDCl}_{3}\right) \delta 7.61(\mathrm{~d}, J=7.3 \mathrm{~Hz}, 2 \mathrm{H}), 7.46(\mathrm{~d}, J=8.4 \mathrm{~Hz}, 2 \mathrm{H}), 7.39-7.28(\mathrm{~m}$, $3 \mathrm{H}), 7.18(\mathrm{~s}, 2 \mathrm{H}), 6.80(\mathrm{~d}, J=8.4 \mathrm{~Hz}, 2 \mathrm{H}), 5.23(\mathrm{~s}, 1 \mathrm{H}), 5.16(\mathrm{~s}, 1 \mathrm{H}), 3.81(\mathrm{~s}, 3 \mathrm{H}), 1.36(\mathrm{~s}, 18 \mathrm{H}) .{ }^{13} \mathrm{C}-\mathrm{NMR}$ $\left(101 \mathrm{MHz}, \mathrm{CDCl}_{3}\right) \delta 154.29$ (s), $137.65(\mathrm{~s}), 136.11$ (s), 133.01 (s), $131.68(\mathrm{~s}), 130.54(\mathrm{~s}), 129.95$ (s), 128.78 (s), $128.65(\mathrm{~s}), 128.53$ (s), $127.08(\mathrm{~s}), 122.89$ (s), 76.97 (s), $34.32(\mathrm{~s}), 30.13$ (s).

N-(4-(((3,5-di-tert-butyl-4-hydroxyphenyl)(phenyl)methyl)sulfonyl)phenyl) acetamide (4ad): Pale yellow solid; 79\% yield. ${ }^{1} \mathrm{H}-\mathrm{NMR}\left(600 \mathrm{MHz}\right.$, DMSO $\left.\left(\mathrm{d}^{6}\right)\right) \delta 10.28(\mathrm{~s}, 1 \mathrm{H}), 7.61(\mathrm{dd}, J=11.6,8.3 \mathrm{~Hz}, 4 \mathrm{H}), 7.46(\mathrm{~d}$, $J=8.7 \mathrm{~Hz}, 2 \mathrm{H}), 7.35(\mathrm{t}, J=7.4 \mathrm{~Hz}, 2 \mathrm{H}), 7.30(\mathrm{t}, J=7.2 \mathrm{~Hz}, 1 \mathrm{H}), 7.23(\mathrm{~s}, 2 \mathrm{H}), 7.03(\mathrm{~s}, 1 \mathrm{H}), 5.77(\mathrm{~s}, 1 \mathrm{H})$, $2.06(\mathrm{~s}, 3 \mathrm{H}), 1.31(\mathrm{~s}, 18 \mathrm{H}) .{ }^{13} \mathrm{C}-\mathrm{NMR}\left(151 \mathrm{MHz}, \mathrm{DMSO}\left(\mathrm{d}^{6}\right)\right) \delta 169.47(\mathrm{~s}), 154.32(\mathrm{~s}), 144.13(\mathrm{~s}), 139.25(\mathrm{~s})$, $134.72(\mathrm{~s}), 130.26(\mathrm{~s}), 130.14(\mathrm{~s}), 128.86(\mathrm{~s}), 128.55$ (s), 126.96 (s), 124.72 (s), $118.24(\mathrm{~s}), 74.46$ (s), 34.96 (s), 30.68 (s), 24.62 (s); HRMS (ESI): $\mathrm{m} / z$ calcd for $\mathrm{C}_{29} \mathrm{H}_{35} \mathrm{NO}_{4} \mathrm{SNa}[\mathrm{M}+\mathrm{Na}]^{+}$, 516.2178; found, 516.2179. 
2,6-Di-tert-butyl-4-(((4-chlorophenyl)sulfonyl)(phenyl)methyl)phenol (4ae): [16] Pale yellow solid; 75\% yield. ${ }^{1} \mathrm{H}-\mathrm{NMR}\left(600 \mathrm{MHz}, \mathrm{DMSO}\left(\mathrm{d}^{6}\right)\right) \delta 7.64(\mathrm{~d}, J=7.3 \mathrm{~Hz}, 2 \mathrm{H}), 7.55(\mathrm{dd}, J=22.6,8.7 \mathrm{~Hz}, 4 \mathrm{H}), 7.39(\mathrm{t}$, $J=7.4 \mathrm{~Hz}, 2 \mathrm{H}), 7.34(\mathrm{t}, J=6.8 \mathrm{~Hz}, 1 \mathrm{H}), 7.21(\mathrm{~s}, 2 \mathrm{H}), 7.08(\mathrm{~s}, 1 \mathrm{H}), 5.95(\mathrm{~s}, 1 \mathrm{H}), 1.31(\mathrm{~s}, 18 \mathrm{H}) .{ }^{13} \mathrm{C}-\mathrm{NMR}$ (151 MHz, DMSO(d $\left.\left.\mathrm{d}^{6}\right)\right) \delta 154.47$ (s), 139.40 (s), 138.99 (s), 137.71 (s), 134.04 (s), 130.83 (s), 130.31 (s), 129.18 (s), $129.00(\mathrm{~s}), 128.78(\mathrm{~s}), 127.05$ (s), 124.18 (s), 74.08 (s), 34.95 (s), 30.63 (s).

4-(((4-Bromophenyl)sulfonyl)(phenyl)methyl)-2,6-di-tert-butylphenol (4af): [16] Pale yellow solid; 81\% yield. ${ }^{1} \mathrm{H}-\mathrm{NMR}\left(400 \mathrm{MHz}, \mathrm{CDCl}_{3}\right) \delta 7.61(\mathrm{dd}, J=7.7,1.8 \mathrm{~Hz}, 2 \mathrm{H}), 7.50-7.45(\mathrm{~m}, 2 \mathrm{H}), 7.42-7.40(\mathrm{~m}, 1 \mathrm{H})$, 7.40-7.32 (m, 4H), $7.17(\mathrm{~s}, 2 \mathrm{H}), 5.26(\mathrm{~s}, 1 \mathrm{H}), 5.17(\mathrm{~s}, 1 \mathrm{H}), 1.37(\mathrm{~s}, 18 \mathrm{H}) .{ }^{13} \mathrm{C}-\mathrm{NMR}\left(101 \mathrm{MHz}, \mathrm{CDCl}_{3}\right) \delta$ $154.29(\mathrm{~s}), 137.65(\mathrm{~s}), 136.11(\mathrm{~s}), 133.01(\mathrm{~s}), 131.68(\mathrm{~s}), 130.54(\mathrm{~s}), 129.95(\mathrm{~s}), 128.78(\mathrm{~s}), 128.65(\mathrm{~s}), 128.53(\mathrm{~s})$, $127.08(\mathrm{~s}), 122.89(\mathrm{~s}), 76.97(\mathrm{~s}), 34.32(\mathrm{~s}), 30.13(\mathrm{~s})$.

2,6-Di-tert-butyl-4-(((4-fluorophenyl)sulfonyl)(phenyl)methyl)phenol (4ag): [16] Pale yellow solid; 62\% yield. ${ }^{1} \mathrm{H}-\mathrm{NMR}\left(600 \mathrm{MHz}, \mathrm{CDCl}_{3}\right) \delta 7.60(\mathrm{dd}, J=7.9,1.2 \mathrm{~Hz}, 2 \mathrm{H}), 7.58-7.53(\mathrm{~m}, 2 \mathrm{H}), 7.38-7.28(\mathrm{~m}, 3 \mathrm{H}), 7.21(\mathrm{~s}$, 2H), 7.04-6.95 (m, 2H), $5.26(\mathrm{~s}, 1 \mathrm{H}), 5.18(\mathrm{~s}, 1 \mathrm{H}), 1.37(\mathrm{~s}, 18 \mathrm{H}) .{ }^{13} \mathrm{C}-\mathrm{NMR}\left(101 \mathrm{MHz}, \mathrm{CDCl}_{3}\right) \delta 164.46(\mathrm{~d}$, $J=255.9 \mathrm{~Hz}), 153.21(\mathrm{~s}), 135.05(\mathrm{~s}), 133.62(\mathrm{~s}), 132.21(\mathrm{~s}), 130.79(\mathrm{~d}, J=9.5 \mathrm{~Hz}), 128.91(\mathrm{~s}), 127.70(\mathrm{~s})$, $127.55(\mathrm{~s}), 126.00(\mathrm{~s}), 122.05(\mathrm{~s}), 114.63(\mathrm{~d}, J=22.5 \mathrm{~Hz}), 75.99(\mathrm{~s}), 33.30(\mathrm{~s}), 29.12(\mathrm{~s})$.

2,6-Di-tert-butyl-4-(phenyl((4-(trifluoromethyl)phenyl)sulfonyl)methyl)phenol (4ah): [16] Pale yellow solid; $82 \%$ yield. ${ }^{1} \mathrm{H}-\mathrm{NMR}\left(600 \mathrm{MHz}, \mathrm{CDCl}_{3}\right) \delta 7.69(\mathrm{~d}, J=8.1 \mathrm{~Hz}, 2 \mathrm{H}), 7.65-7.57(\mathrm{~m}, 4 \mathrm{H}), 7.40-7.33(\mathrm{~m}$, 3H), $7.17(\mathrm{~s}, 2 \mathrm{H}), 5.27(\mathrm{~s}, 1 \mathrm{H}), 5.21(\mathrm{~s}, 1 \mathrm{H}), 1.35(\mathrm{~s}, 18 \mathrm{H}) .{ }^{13} \mathrm{C}-\mathrm{NMR}\left(151 \mathrm{MHz}, \mathrm{DMSO}\left(\mathrm{d}^{6}\right)\right) \delta 154.68(\mathrm{~s})$, $142.95(\mathrm{~s}), 139.58(\mathrm{~s}), 134.06-133.29(\mathrm{~m}), 133.82(\mathrm{~s}), 130.51(\mathrm{~s}), 130.13(\mathrm{~s}), 129.22(\mathrm{~s}), 129.06(\mathrm{~s}), 127.28(\mathrm{~s})$, 126.44-126.15 (m), $123.96(\mathrm{q}, J=273.0 \mathrm{~Hz}), 123.98(\mathrm{~s}), 74.06(\mathrm{~s}), 35.07(\mathrm{~s}), 30.72(\mathrm{~s})$.

2,6-Di-tert-butyl-4-(phenyl((4-(trifluoromethoxy)phenyl)sulfonyl)methyl)phenol (4ai): Pale yellow solid; 84\% yield. ${ }^{1} \mathrm{H}-\mathrm{NMR}\left(600 \mathrm{MHz}, \mathrm{CDCl}_{3}\right) \delta 7.61(\mathrm{~d}, J=6.8 \mathrm{~Hz}, 1 \mathrm{H}), 7.48(\mathrm{~d}, J=8.5 \mathrm{~Hz}, 1 \mathrm{H}), 7.37-7.32(\mathrm{~m}$, $1 \mathrm{H}), 7.31(\mathrm{~d}, J=8.5 \mathrm{~Hz}, 1 \mathrm{H}), 7.18(\mathrm{~s}, 1 \mathrm{H}), 5.25(\mathrm{~s}, 1 \mathrm{H}), 5.17(\mathrm{~s}, 1 \mathrm{H}), 1.37(\mathrm{~s}, 1 \mathrm{H}) .{ }^{13} \mathrm{C}-\mathrm{NMR}(151 \mathrm{MHz}$, $\left.\operatorname{DMSO}\left(\mathrm{d}^{6}\right)\right) \delta 154.48(\mathrm{~s}), 152.04(\mathrm{~d}, J=2.0 \mathrm{~Hz}), 139.39(\mathrm{~s}), 137.73(\mathrm{~s}), 134.00(\mathrm{~s}), 131.65(\mathrm{~s}), 130.32(\mathrm{~s})$, 129.00 (s), 128.80 (s), 127.04 (s), 124.13 (s), 121.18 (s), 120.26 (q, J = 258.2 Hz).74.07 (s), 34.93 (s), 30.59 (s); HRMS (ESI): $m / z$ calcd for $\mathrm{C}_{28} \mathrm{H}_{31} \mathrm{~F}_{3} \mathrm{O}_{4} \mathrm{SNa}[\mathrm{M}+\mathrm{Na}]^{+}, 543.1785$; found, 543.1787 .

2,6-Di-tert-butyl-4-(((3-nitrophenyl)sulfonyl)(phenyl)methyl)phenol (4aj): Pale yellow solid; 86\% yield. ${ }^{1} \mathrm{H}-\mathrm{NMR}\left(600 \mathrm{MHz}, \mathrm{CDCl}_{3}\right) \delta 8.38-8.32(\mathrm{~m}, 1 \mathrm{H}), 8.30(\mathrm{t}, J=1.7 \mathrm{~Hz}, 1 \mathrm{H}), 7.94(\mathrm{~d}, J=7.7 \mathrm{~Hz}, 1 \mathrm{H}), 7.63(\mathrm{~d}$, $J=6.6 \mathrm{~Hz}, 2 \mathrm{H}), 7.58(\mathrm{t}, J=8.0 \mathrm{~Hz}, 1 \mathrm{H}), 7.42-7.33(\mathrm{~m}, 3 \mathrm{H}), 7.23(\mathrm{~s}, 2 \mathrm{H}), 5.30(\mathrm{~s}, 1 \mathrm{H}), 5.24(\mathrm{~s}, 1 \mathrm{H}), 1.36(\mathrm{~s}$, 18H). ${ }^{13} \mathrm{C}-\mathrm{NMR}\left(151 \mathrm{MHz}, \mathrm{CDCl}_{3}\right) \delta 154.56$ (s), 147.81 (s), 140.94 (s), 136.49 (s), 134.26 (s), 132.33 (s), 129.99 (s), $129.66(\mathrm{~s}), 128.97$ (s), $128.93(\mathrm{~s}), 127.49$ (s), 127.07 (s), 124.48 (s), 122.32 (s), 77.12 (s), 34.35 (s), 30.14 (s); HRMS (ESI): $\mathrm{m} / z$ calcd for $\mathrm{C}_{27} \mathrm{H}_{31} \mathrm{NO}_{5} \mathrm{SNa}[\mathrm{M}+\mathrm{Na}]^{+}$, 504.1817; found, 504.1815.

2,6-Di-tert-butyl-4-((naphthalen-1-ylsulfonyl)(phenyl)methyl)phenol (4ak): [16] Pale yellow solid; 52\% yield. ${ }^{1} \mathrm{H}-\mathrm{NMR}\left(400 \mathrm{MHz}, \mathrm{CDCl}_{3}\right) \delta 8.07(\mathrm{~d}, J=1.2 \mathrm{~Hz}, 1 \mathrm{H}), 7.85(\mathrm{~d}, J=8.2 \mathrm{~Hz}, 1 \mathrm{H}), 7.79(\mathrm{dd}, J=8.1,5.2 \mathrm{~Hz}$, $2 \mathrm{H}), 7.67(\mathrm{dd}, J=7.8,1.6 \mathrm{~Hz}, 2 \mathrm{H}), 7.64-7.51(\mathrm{~m}, 3 \mathrm{H}), 7.39-7.30(\mathrm{~m}, 3 \mathrm{H}), 7.16(\mathrm{~s}, 2 \mathrm{H}), 5.28(\mathrm{~s}, 1 \mathrm{H}), 5.18(\mathrm{~s}$, 1H), $1.26(\mathrm{~s}, 18 \mathrm{H}) .{ }^{13} \mathrm{C}-\mathrm{NMR}\left(101 \mathrm{MHz}, \mathrm{CDCl}_{3}\right) \delta 153.06(\mathrm{~s}), 134.88(\mathrm{~s}), 134.37$ (s), 133.97 (s), 132.31 (s), $130.81(\mathrm{~s}), 130.02(\mathrm{~s}), 129.01(\mathrm{~s}), 128.23(\mathrm{~s}), 128.02(\mathrm{~s}), 127.67(\mathrm{~s}), 127.47(\mathrm{~s}), 127.42(\mathrm{~s}), 126.74(\mathrm{~s}), 126.34(\mathrm{~s})$, $126.04(\mathrm{~s}), 122.65(\mathrm{~s}), 122.38(\mathrm{~s}), 75.89(\mathrm{~s}), 33.18(\mathrm{~s}), 29.01(\mathrm{~s})$.

2,6-Di-tert-butyl-4-(phenyl(thiophen-2-ylsulfonyl)methyl)phenol (4al): [16] Pale yellow solid; 76\% yield. ${ }^{1} \mathrm{H}-\mathrm{NMR}\left(600 \mathrm{MHz}, \mathrm{CDCl}_{3}\right) \delta 7.63(\mathrm{~d}, J=7.0 \mathrm{~Hz}, 2 \mathrm{H}), 7.54(\mathrm{~d}, J=3.7 \mathrm{~Hz}, 1 \mathrm{H}), 7.40-7.30(\mathrm{~m}, 3 \mathrm{H}), 7.28$ $(\mathrm{s}, 3 \mathrm{H}), 7.01-6.89(\mathrm{~m}, 1 \mathrm{H}), 5.30(\mathrm{~s}, 1 \mathrm{H}), 5.26(\mathrm{~s}, 1 \mathrm{H}), 1.38(\mathrm{~s}, 18 \mathrm{H}) .{ }^{13} \mathrm{C}-\mathrm{NMR}\left(151 \mathrm{MHz}, \mathrm{DMSO}\left(\mathrm{d}^{6}\right)\right) \delta$ $154.54(\mathrm{~s}), 139.54(\mathrm{~s}), 139.40(\mathrm{~s}), 136.07(\mathrm{~s}), 135.32(\mathrm{~s}), 134.54(\mathrm{~s}), 130.23(\mathrm{~s}), 128.97(\mathrm{~s}), 128.78(\mathrm{~s}), 128.05(\mathrm{~s})$, $126.89(\mathrm{~s}), 124.58(\mathrm{~s}), 75.79(\mathrm{~s}), 35.02(\mathrm{~s}), 30.71(\mathrm{~s})$.

4-((Benzylsulfonyl)(phenyl)methyl)-2,6-di-tert-butylphenol (4am): [16] Pale yellow solid; 84\% yield. ${ }^{1} \mathrm{H}-\mathrm{NMR}\left(600 \mathrm{MHz}, \mathrm{CDCl}_{3}\right) \delta 7.57(\mathrm{dd}, J=5.2,3.3 \mathrm{~Hz}, 2 \mathrm{H}), 7.42-7.29(\mathrm{~m}, 8 \mathrm{H}), 7.17(\mathrm{dd}, J=7.9$, $1.3 \mathrm{~Hz}, 2 \mathrm{H}), 5.31(\mathrm{~s}, 1 \mathrm{H}), 5.03(\mathrm{~s}, 1 \mathrm{H}), 4.33-3.57(\mathrm{~m}, 2 \mathrm{H}), 1.44(\mathrm{~s}, 18 \mathrm{H}) .{ }^{13} \mathrm{C}-\mathrm{NMR}\left(101 \mathrm{MHz}, \mathrm{CDCl}_{3}\right) \delta$ 
$154.38(\mathrm{~s}), 136.32(\mathrm{~s}), 132.83(\mathrm{~s}), 130.97(\mathrm{~s}), 130.11(\mathrm{~s}), 128.82(\mathrm{~s}), 128.78(\mathrm{~s}), 128.68(\mathrm{~d}, J=2.0 \mathrm{~Hz}), 128.07$ (s), $126.88(\mathrm{~s}), 122.93(\mathrm{~s}), 71.81(\mathrm{~s}), 58.13(\mathrm{~s}), 34.48(\mathrm{~s}), 30.30(\mathrm{~s})$.

3.2.2. Representative Procedure for General Reaction Procedure for Synthesis of Unsymmetrical Triarylmethane 7

To a solution of 2,6-di-tert-butyl-4-(phenyl(tosyl)methyl) phenol ( $45 \mathrm{mg}, 0.10 \mathrm{mmol}$ ) in $1 \mathrm{~mL} \mathrm{DCE}$, $\mathrm{KOH}(5.6 \mathrm{mg}, 0.10 \mathrm{mmol})$ and indole $(14.06 \mathrm{mg}, 0.12 \mathrm{mmol})$ were added. The mixture was stirred at $65^{\circ} \mathrm{C}$. The reaction was monitored by TLC. After complete reaction, the mixture was washed with water, dried over by anhydrous magnesium sulfate, and concentrated in vacuo. The crude product was then purified by flash column chromatography on silica gel to afford the pure product 7 .

2,6-Di-tert-butyl-4-((3a,7a-dihydro-1H-indol-3-yl)(phenyl)methyl)phenol (7): [41] Brown solid; 85\% yield. ${ }^{1} \mathrm{H}-\mathrm{NMR}\left(600 \mathrm{MHz}, \mathrm{CDCl}_{3}\right) \delta 7.90(\mathrm{~s}, 1 \mathrm{H}), 7.33(\mathrm{~d}, J=8.1 \mathrm{~Hz}, 1 \mathrm{H}), 7.25(\mathrm{~d}, J=5.3 \mathrm{~Hz}, 4 \mathrm{H}), 7.22(\mathrm{~d}$, $J=8.1 \mathrm{~Hz}, 1 \mathrm{H}), 7.18(\mathrm{qd}, J=5.3,2.8 \mathrm{~Hz}, 1 \mathrm{H}), 7.14(\mathrm{t}, J=7.5 \mathrm{~Hz}, 1 \mathrm{H}), 7.04(\mathrm{~s}, 2 \mathrm{H}), 6.97(\mathrm{t}, J=7.5 \mathrm{~Hz}, 1 \mathrm{H})$, $6.58(\mathrm{~d}, J=23.9 \mathrm{~Hz}, 1 \mathrm{H}), 5.56(\mathrm{~s}, 1 \mathrm{H}), 5.04(\mathrm{~s}, 1 \mathrm{H}), 1.36(\mathrm{~s}, 18 \mathrm{H}) \cdot{ }^{13} \mathrm{C}-\mathrm{NMR}\left(151 \mathrm{MHz}, \mathrm{CDCl}_{3}\right) \delta 152.91$ $(\mathrm{d}, J=2.9 \mathrm{~Hz}), 141.67(\mathrm{~s}), 136.43(\mathrm{~d}, J=2.7 \mathrm{~Hz}), 135.80(\mathrm{~d}, J=2.2 \mathrm{~Hz}), 131.54(\mathrm{~s}), 131.31(\mathrm{~s}), 128.61(\mathrm{~d}$, $J=2.9 \mathrm{~Hz}), 128.43(\mathrm{~s}), 127.04(\mathrm{~d}, J=2.6 \mathrm{~Hz}), 126.61(\mathrm{~d}, J=3.0 \mathrm{~Hz}), 125.21(\mathrm{~d}, J=2.8 \mathrm{~Hz}), 58.10(\mathrm{~s}), 34.43$ (s), $30.33(\mathrm{~s})$.

3.2.3. Representative Procedure for General Reaction Procedure for Synthesis of Unsymmetrical Gem-Diarylmethyl Thioether 8

To a solution of 2,6-di-tert-butyl-4-(phenyl(tosyl)methyl) phenol ( $45 \mathrm{mg}, 0.1 \mathrm{mmol}$ ) in $1 \mathrm{~mL}$ methyl tert-butyl ether, $\mathrm{Cs}_{2} \mathrm{CO}_{3}(36 \mathrm{mg}, 0.01 \mathrm{mmol})$ and TBAB $(3.6 \mathrm{mg}, 0.01 \mathrm{mmol})$ were added. Benzenethiol $(13.68 \mu \mathrm{L}, 0.12 \mathrm{mmol})$ was added to the solution dropwise, and the mixture was stirred at $80^{\circ} \mathrm{C}$. The reaction was monitored by TLC. After complete reaction, the mixture was concentrated in vacuo. The crude product was then purified by flash column chromatography on silica gel to afford the pure product 8.

2,6-Di-tert-butyl-4-(phenyl(phenylthio)methyl)phenol (8): [42] Colorless gummy liquid; 76\% yield. ${ }^{1} \mathrm{H}-\mathrm{NMR}$ $(600 \mathrm{MHz}, \mathrm{CDCl} 3) \delta 7.38(\mathrm{~d}, J=7.4 \mathrm{~Hz}, 2 \mathrm{H}), 7.20(\mathrm{t}, J=7.3 \mathrm{~Hz}, 2 \mathrm{H}), 7.12(\mathrm{t}, J=7.0 \mathrm{~Hz}, 3 \mathrm{H}), 7.10-7.00(\mathrm{~m}$, $5 \mathrm{H}), 5.35(\mathrm{~d}, J=23.3 \mathrm{~Hz}, 1 \mathrm{H}), 5.01(\mathrm{~d}, J=23.1 \mathrm{~Hz}, 1 \mathrm{H}), 1.29(\mathrm{~s}, 18 \mathrm{H}) .{ }^{13} \mathrm{C}-\mathrm{NMR}(151 \mathrm{MHz}, \mathrm{CDCl} 3) \delta$ $152.00(\mathrm{~s}), 144.65(\mathrm{~s}), 136.75(\mathrm{~s}), 135.42(\mathrm{~s}), 135.29$ (s), $134.46(\mathrm{~s}), 128.95(\mathrm{~s}), 128.09$ (s), 127.19 (s), $125.92(\mathrm{~s})$, $125.54(\mathrm{~s}), 123.80$ (s), 121.92 (s), 120.11 (s), 119.23 (s), 110.89 (s), 48.84 (s), 34.35 (s), 30.40 (s).

\section{Conclusions}

In summary, we developed the TBAB-promoted sulfa-1,6-conjugated addition of para-quinone methides with sulfonyl hydrazines in water. This reaction provides a green and sustainable method for direct synthesis of various unsymmetrical diarylmethyl sulfones with good functional group tolerance, scalability, and regioselectivity. Further transformation of the resulting diarylmethyl sulfones provides an efficient route to some functionalized molecules.

Supplementary Materials: The following are available online, the ${ }^{1} \mathrm{H}-\mathrm{NMR},{ }^{13} \mathrm{C}-\mathrm{NMR}$ spectra of compounds 3aa-3ay and 4aa-4am, 7, 8; HRMS data of 4ad, 4ai and 4aj; crystal data of 4af are available as supporting data.

Author Contributions: Z.-Q.L. proposed the subject and designed the study, P.-S.Y. and D.-Q.L. carried out the chemical experiments, L.-D.Z. and S.-S.L. performed electrochemical data analysis, and X.-Y.G. wrote the article. All authors have read and agreed to the published version of the manuscript.

Funding: This work was supported by the National Natural Science Foundation of China (Grant No. 21402159), the Fundamental Research Funds for the Central Universities (XDJK2019AA003), the Chongqing Postdoctoral Science Foundation (Xm2016110).

Conflicts of Interest: The authors declare no conflict of interest. 


$\begin{array}{ll}\text { Abbreviations } \\ \text { THF } & \text { Tetrahydrofuran } \\ \text { DCE } & \text { Dichloroethane } \\ \text { DMSO } & \text { Dimethylsulfoxide } \\ \text { CTAB } & \text { Cetyltrimethyl Ammonium Bromide } \\ \text { TBAI } & \text { Tetrabutyl Ammonium Iodide } \\ \text { TEBAC } & \text { Triethylbenzyl Ammonium Chloride } \\ \text { TBAB } & \text { Tetrabutyl Ammonium Bromide } \\ \text { SDS } & \text { Sodium Dodecyl Sulfate } \\ \text { HRMS } & \text { High Resolution Mass Spectroscopy }\end{array}$

\section{References}

1. Simpkins, N.S. Sulphones in Organic Synthesis; Pergamon Press: Oxford, UK, 1993.

2. Liang, S.; Shaaban, S.; Liu, N.-W.; Hofman, K.; Manolikakes, G. Chapter Three-Recent Advances in the Synthesis of C-S Bonds via Metal-Catalyzed or -Mediated Functionalization of C-H Bonds. In Advances in Organometallic Chemistry; Pérez, P.J., Ed.; Academic Press: San Diego, CA, USA, 2018; Volume 69, pp. $135-207$.

3. Hofman, K.; Liu, N.-W.; Manolikakes, G. Radicals and sulfur dioxide: A versatile combination for the construction of sulfonyl-containing molecules. Chem. Eur. J. 2018, 24, 11852-11863. [CrossRef] [PubMed]

4. Román, R.; Barrio, P.; Mateu, N.; Sedgwick, D.M.; Fustero, S. Copper-catalyzed regioselective synthesis of (E)- $\beta$-fluorovinyl sulfones. Molecules 2019, 24, 1569.

5. Orooji, Y.; Ghasali, E.; Emami, N.; Noorisafa, F.; Razmjou, A. ANOVA Design for the optimization of $\mathrm{TiO}_{2}$ coating on polyether sulfone membranes. Molecules 2019, 24, 2924. [CrossRef] [PubMed]

6. Alsaedi, A.M.R.; Farghaly, T.A.; Shaaban, M.R. Synthesis and antimicrobial evaluation of novel pyrazolopyrimidines incorporated with mono- and diphenylsulfonyl groups. Molecules 2019, $24,4009$. [CrossRef]

7. Cohen, A.; Crozet, M.D.; Rathelot, P.; Azas, N.; Vanelle, P. Synthesis and promising in vitro antiproliferative activity of sulfones of a 5-nitrothiazole series. Molecules 2013, 18, 97-113. [CrossRef]

8. Gouliaev, A.H.; Slok, F.A.; Teuber, L.; Demnitz, J. Potassium Channel Modulators. U.S. Patent 742,961,8B2, 30 September 2008.

9. Langler, R.F.; Paddock, R.L.; Thompson, D.B.; Crandall, I.; Ciach, M.; Kain, K.C. Selected sulfonyl compounds as anticancer/antimalarial agents. Aust. J. Chem. 2003, 56, 1127-1133. [CrossRef]

10. Ito, N.; Kurimura, M.; Yamauchi, T.; Segawa, C.; Sasaki, H.; Tai, K.; Arai, K.; Shinohara, T. Substituted benzo[1,4]diazepine Derivatives as Antidepressants and Their Preparation. WO Patent WO2009145357A1, 3 November 2009.

11. Schoenebeck, F.; Murphy, J.A.; Zhou, S.-Z.; Uenoyama, Y.; Miclo, Y.; Tuttle, T. Reductive cleavage of sulfones and sulfonamides by a neutral organic super-electron-donor (S.E.D.) reagent. J. Am. Chem. Soc. 2007, 129, 13368-13369. [CrossRef]

12. Nambo, M.; Ariki, Z.T.; Canseco-Gonzalez, D.; Beattie, D.D.; Crudden, C.M. Arylative desulfonation of diarylmethyl phenyl sulfone with arenes catalyzed by scandium triflate. Org. Lett. 2016, 18, 2339-2342. [CrossRef]

13. Nambo, M.; Crudden, C.M. Modular synthesis of triarylmethanes through palladium-catalyzed sequential arylation of methyl phenyl sulfone. Angew. Chem., Int. Ed. 2014, 53, 742-746. [CrossRef]

14. Meyers, C.Y.; Chan-Yu-King, R.; Hua, D.H.; Kolb, V.M.; Matthews, W.S.; Parady, T.E.; Horii, T.; Sandrock, P.B.; Hou, Y.; Xie, S. Unexpected differences in the $\alpha$-halogenation and related reactivity of sulfones with perhaloalkanes in KOH-t-BuOH. J. Org. Chem. 2003, 68, 500-511. [CrossRef]

15. Guan, X.-Y.; Zhang, L.-D.; You, P.-S.; Liu, S.-S.; Liu, Z.-Q. 1,6-Conjugate sulfonylation of para-quinone methides: An expedient approach to unsymmetrical gem-diarylmethyl sulfones. Tetrahedron Lett. 2019, 60, 244-247. [CrossRef]

16. Liu, T.; Liu, J.; Xia, S.; Meng, J.; Shen, X.; Zhu, X.; Chen, W.; Sun, C.; Cheng, F. Catalyst-free 1,6-conjugate addition/aromatization/aulfonylation of para-quinone methides: Facile access to diarylmethyl sulfones. ACS Omega 2018, 3, 1409-1415. [CrossRef] [PubMed] 
17. Reddy, M.A.; Reddy, P.S.; Sreedhar, B. Iron(III) chloride-catalyzed direct sulfonylation of alcohols with sodium arenesulfinates. Adv. Synth. Catal. 2010, 352, 1861-1869. [CrossRef]

18. Feng, X.-W.; Wang, J.; Zhang, J.; Yang, J.; Wang, N.; Yu, X.-Q. Copper-catalyzed nitrogen loss of Sulfonylhydrazones: A reductive strategy for the synthesis of sulfones from carbonyl compounds. Org. Lett. 2010, 12, 4408-4411. [CrossRef]

19. Zhu, X.; Li, W.; Luo, X.; Deng, G.; Liang, Y.; Liu, J. A catalyst-free and additive-free method for the synthesis of benzothiazolethiones from o-iodoanilines, DMSO and potassium sulfide. Green Chem. 2018, 20, 1970-1974. [CrossRef]

20. Qu, R.; Zhang, W.; Liu, N.; Zhang, Q.; Liu, Y.; Li, X.; Wei, Y.; Feng, L. Antioil $\mathrm{Ag}_{3} \mathrm{PO}_{4}$ nanoparticle/polydopamine/ $\mathrm{Al}_{2} \mathrm{O}_{3}$ sandwich structure for complex wastewater treatment: Dynamic catalysis under natural light. ACS Sustainable Chem. Eng. 2018, 6, 8019-8028. [CrossRef]

21. Bhaumik, P.; Chou, H.-J.; Lee, L.-C.; Chung, P.-W. Chemical transformation for 5-hydroxymethylfurfural production from saccharides using molten salt system. ACS Sustainable Chem. Eng. 2018, 6, 5712-5717. [CrossRef]

22. Ishizuka, T.; Ohkawa, S.; Ochiai, H.; Hashimoto, M.; Ohkubo, K.; Kotani, H.; Sadakane, M.; Fukuzumi, S.; Kojima, T. A supramolecular photocatalyst composed of a polyoxometalate and a photosensitizing water-soluble porphyrin diacid for the oxidation of organic substrates in water. Green Chem. 2018, 20, 1975-1980. [CrossRef]

23. Teng, Q.-H.; Peng, X.-J.; Mo, Z.-Y.; Xu, Y.-L.; Tang, H.-T.; Wang, H.-S.; Sun, H.-B.; Pan, Y.-M. Transition-metal-free C-N and C-C formation: Synthesis of benzo [4,5] imidazo [1-a] pyridines and 2-pyridones from ynones. Green Chem. 2018, 20, 2007-2012. [CrossRef]

24. Narayan, S.; Muldoon, J.; Finn, M.G.; Fokin, V.V.; Kolb, H.C.; Sharpless, K.B. “On water”: Unique reactivity of organic compounds in aqueous suspension. Angew. Chem. Int. Ed. 2005, 44, 3275-3279. [CrossRef]

25. Chanda, A.; Fokin, V.V. Organic synthesis "on water". Chem. Rev. 2009, 109, 725-748. [CrossRef] [PubMed]

26. Butler, R.N.; Coyne, A.G. Water: Nature's reaction enforcer-comparative effects for organic synthesis "in-water" and "on-water". Chem. Rev. 2010, 110, 6302-6337. [CrossRef] [PubMed]

27. Lindström, U.M. Stereoselective organic reactions in water. Chem. Rev. 2002, 102, 2751-2772. [CrossRef] [PubMed]

28. Carril, M.; SanMartin, R.; Tellitu, I.; Domínguez, E. On-water chemistry: copper-catalyzed straightforward synthesis of benzo[b]furan derivatives in neat water. Org. Lett. 2006, 8, 1467-1470. [CrossRef] [PubMed]

29. Ndiaye, D.; Coufourier, S.; Mbaye, M.D.; Gaillard, S.; Renaud, J.-L. Cyclopentadienone iron tricarbonyl complexes-catalyzed hydrogen transfer in water. Molecules 2020, 25, 421. [CrossRef] [PubMed]

30. Ghosh, S.; Saha, S.; Sengupta, D.; Chattopadhyay, S.; De, G.; Basu, B. Stabilized $\mathrm{Cu}_{2} \mathrm{O}$ nanoparticles on macroporous polystyrene resins [ $\left.\mathrm{Cu}_{2} \mathrm{O} @ \mathrm{ARF}\right]$ : Improved and reusable heterogeneous catalyst for on-water synthesis of triazoles via click reaction. Ind. Eng. Chem. Res. 2017, 56, 11726-11733. [CrossRef]

31. Pesnot, T.; Gershater, M.C.; Edwards, M.; Ward, J.M.; Hailes, H.C. One-pot phosphate-mediated synthesis of novel 1,3,5-trisubstituted pyridinium salts: A new family of S. aureus inhibitors. Molecules 2017, 22, 626. [CrossRef]

32. Hajra, S.; Singha Roy, S.; Aziz, S.M.; Das, D. Catalyst-Free “On-Water” Regio- and stereospecific ring-opening of spiroaziridine oxindole: Enantiopure synthesis of unsymmetrical 3,3'-bisindoles. Org. Lett. 2017, 19, 4082-4085. [CrossRef]

33. Ibacache, J.A.; Valderrama, J.A.; Faúndes, J.; Danimann, A.; Recio, F.J.; Zúñiga, C.A. Green synthesis and electrochemical properties of mono- and dimers derived from phenylaminoisoquinolinequinones. Molecules 2019, 24, 4378. [CrossRef]

34. Rideout, D.C.; Breslow, R. Hydrophobic acceleration of Diels-Alder reactions. J. Am. Chem. Soc. 1980, 102, 7816-7817. [CrossRef]

35. Denes, F.; Pichowicz, M.; Povie, G.; Renaud, P. Thiyl radicals in organic synthesis. Chem. Rev. 2014, 114, 2587-2693. [CrossRef] [PubMed]

36. Osako, T.; Ueno, Y.; Tachi, Y.; Itoh, S. C-S bond formation reaction between a phenolate and disulfide-bridged dicopper (I) complexes. Inorg. Chem. 2004, 43, 6516-6518. [CrossRef] [PubMed]

37. Ghosh, A.K.; Osswald, H.L.; Prato, G. Recent progress in the development of HIV-1 protease inhibitors for the treatment of HIV/AIDS. J. Med. Chem. 2016, 59, 5172-5208. [CrossRef] [PubMed] 
38. Gao, G.-Y.; Colvin, A.J.; Chen, Y.; Zhang, X.P. Synthesis of meso-arylsulfanyl-and alkylsulfanyl-substituted porphyrins via palladium-mediated C-S bond formation. J. Org. Chem. 2004, 69, 8886-8892. [CrossRef] [PubMed]

39. Liu, Z.-Q.; Guan, X.-Y.; Liu, S.-S.; Ma, X.-B.; Li, Y.-Q.; Zhang, L.-D. Preparation of gem-diarylmethyl Amines Derivatives. China Patent CN201710847133, 28 May 2019.

40. Liu, Z.-Q.; Zhang, L.-D.; Ma, X.-B.; Li, Y.-Q.; Guan, X.-Y. Preparation of gem-diarylmethyl Sulfones in Water. China Patent CN201710848209, 18 January 2019.

41. Gao, S.; Xu, X.; Yuan, Z.; Zhou, H.; Yao, H.; Lin, A. 1,6-Addition arylation of para-quinone methides: An approach to unsymmetrical triarylmethanes. Eur. J. Org. Chem. 2016, 17, 3006-3012. [CrossRef]

42. Jadhav, A.S.; Anand, R.V. Triflic acid catalyzed 1,6-conjugate addition of thiols to $p$-quinone methides under continuous-flow conditions. Eur. J. Org. Chem. 2017, 25, 3716-3721. [CrossRef]

Sample Availability: Samples of the compounds compounds 3aa-3ay and 4aa-4am are available from the authors.

(C) 2020 by the authors. Licensee MDPI, Basel, Switzerland. This article is an open access article distributed under the terms and conditions of the Creative Commons Attribution (CC BY) license (http://creativecommons.org/licenses/by/4.0/). 